\title{
Distribution Network Design on the Battlefield
}

\author{
Hunkar Toyoglu, Oya Ekin Karasan, Bahar Yetis Kara \\ Department of Industrial Engineering, Bilkent University, Ankara 06800, Turkey \\ Received 12 April 2007; revised 19 December 2009; accepted 30 December 2009 \\ DOI 10.1002/nav.20402 \\ Published online 29 April 2010 in Wiley Online Library (wileyonlinelibrary.com).
}

\begin{abstract}
Ammunition, whether it be an arrow in the middle ages, a lead bullet in the 1800s, or a laser guided smart bomb today, has been the most prominent factor in determining the outcome of combat. Failing to supply the required amount of ammunition properly may lead to defeat. Our main objective in this study is to provide a decision support tool that can help plan ammunition distribution on the battlefield. We demonstrate through an extensive literature review that the existing models are not capable of handling the specifics of the problem in this study. To this end, we propose a novel three-layer commodity-flow location routing formulation that distributes multiple products, respects hard time windows, allows demand points to be supplied by more than one vehicle or depot, and locates facilities at two different layers. We derive several valid inequalities to speed up the solution time of our model, illustrate the performance of the model in several realistically sized scenarios, and report encouraging results. Finally, we introduce a dynamic model that designs the distribution system in consecutive time periods for the entire combat duration. (C) 2010 Wiley Periodicals, Inc. Naval Research Logistics 58: 188-209, 2011
\end{abstract}

Keywords: military application; logistics; location routing problem; network design

\section{INTRODUCTION AND MOTIVATION}

The success of a combat operation depends on the availability of ammunition (henceforth called ammo), because a combat unit can only fight if it receives ammo in the proper quantity, when and where it is needed. Therefore, ammo is the dominant factor in determining the outcome of combat, and any failure to supply the required amount of it may result in tactical defeat.

Previously, the land forces of most countries relied on heavy forces that were equipped with a large number of heavy weapons for their lethality. More information about the changing of land forces over the years can be found from [68] by searching for the history of any specific army. The more heavy weapons a land force has, the more fire power it has, and the more lethal it is. However, when equipped with such numbers and types of weapons, land forces lose their ability to move fast, and they need to keep enormous ammo inventories stocked at huge depots. Hence, the ammo distribution system of these land forces is designed to support a heavy and slow moving force. It usually consists of different types of depots, most of which are underground storage facilities, bunkers, or fortified storage areas. In this system, ammo is pushed down from higher level depots to lower level depots, and

Correspondence to: H. Toyoglu (hunkar@bilkent.edu.tr) combat units pull their ammo requirements from the lowest level depots with their own trucks.

With the end of Cold War, most of these traditional heavy land forces moved towards a smaller, more agile, and deployable force. For more information, see [68] and search for the transformation of any specific army. Such a force does not depend solely on its firepower, but also on its mobility. This characteristic enables newly structured forces to move further and faster on the battlefield. To supply such a fast moving force, an effective and efficient distribution system is needed.

We propose a Mobile Ammunition Distribution System (Mobile-ADS) to satisfy this requirement. Our main objective is to deliver ammo as close to the combat units as possible and do this in a timely manner. To do so, we suggest Fixed Transfer Points (Fixed-TPs) and Mobile Transfer Points (Mobile-TPs), that — after proper positioning — will reduce the need for the remaining depots. Fixed-TPs are either railheads where a rail network ends or suitable locations on a rail network where ammo can be transported safely as far as possible. Ammo is transferred from trains to commercial trucks at Fixed-TPs. Mobile-TPs are mostly forward staging areas where ammo trucks or small stocks of ammo are kept for a short period of time before being moved further forward to support front line combat units. They are located as close to combat units as possible to provide the shortest supply time. Ammo is transferred from commercial trucks to ammo trucks at Mobile-TPs. Figure 1 shows an example of a 


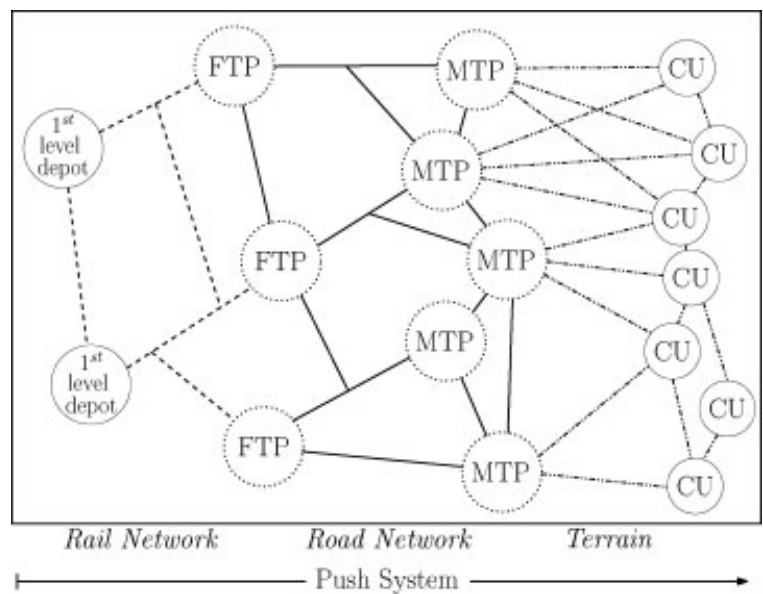

Figure 1. Mobile ammo distribution system on the battlefield.

Mobile-ADS on the battlefield. Solid (dotted) circles represent fixed (potential) locations. In this figure, first level depot, FTP, MTP, and CU denote highest level depots, Fixed-TPs, Mobile-TPs, and combat units, respectively.

A combat environment can change rapidly. Unexpected strategic or tactical developments may unfold and can render earlier logistics support plans obsolete. In such a rapidly changing and hostile environment, Mobile-TPs, with their small and mobile structure, can support combat units by moving with them accordingly.

The problem of designing Mobile-ADS can be very complex if we want to capture all realities at once. Since battles generally continue for days, weeks, or months, the problem in its entirety has a challenging dynamic nature, which requires the consideration of several consecutive planning periods. A typical long-term strategic plan involving several planning periods happens long before the actual war commences. Hence, solution time may not be of major concern during long-term strategic planning. However, solution time is of great importance during tactical decision making process, since such decisions, which are related to a single planning period, must be made in a very short time. In view of this, we present both the single and multi planning period versions of our problem.

The rest of this article is organized as follows: Section 2 describes the Mobile-ADS design problem, reviews the literature, and compares the characteristics of our problem with those of the majority of the literature. Section 3 demonstrates the mathematical formulation of the static design problem and derives several valid inequalities. Section 4 analyzes the effectiveness of the valid inequalities in some test problem instances and then exhibits the performance of the model in several realistically sized scenarios. Section 5 introduces the dynamic design problem and gives its mathematical formulation. Section 6 shows how the static model could be used in a combat environment for a multi-period operation and highlights how the model could be utilized in assisting the logistics planners when faced with unplanned combat situations. The summary and the conclusions are presented in Section 7.

\section{PROBLEM DEFINITION AND RELATED LITERATURE}

In this section, we first define the Mobile-ADS design problem. We then develop a classification scheme and classify 78 articles from the literature. Finally, we give the classification of our problem and highlight how it differs from the existing studies in the literature.

\subsection{Problem Definition}

Mobile-ADS is a continuous replenishment and true push system. Highest level depots are the first to receive ammo that is produced or procured. Ammo is then moved to FixedTPs on rail networks. Within the context of the current study, we do not analyze the flow from the highest level depots to Fixed-TPs. We assume that the required amount of ammo can be carried in between by rail, on time.

Ammo is moved from Fixed-TPs to Mobile-TPs by commercial trucks on road networks. Then, Mobile-TPs issue ammo to combat units with ammo trucks that have the capability to move off-road and to load and unload themselves with their own cranes. In such a system, combat units will not take on the logistic burden of pulling their own ammo.

Consider a battlefield containing Fixed-TPs, Mobile-TPs, and combat units. We need to decide the locations of FixedTPs and Mobile-TPs to provide a bridge between the highest level depots and combat units, and the truck routes and schedules to distribute ammo from Fixed-TPs to combat units via Mobile-TPs. Solving these two problems separately may lead to suboptimal decisions (see, e.g., [75]). Therefore, these decisions must be made simultaneously. Hence, the MobileADS design problem that combines location, routing, and scheduling into a single model is a Location Routing Problem (LRP).

\subsection{Literature Review}

Several earlier studies introduce the LRP or review LRP literature (see, e.g., [10], [40], [52], [58] and [63]). In the two most recent studies, [58] used 12, and [63] used nine (both including the solution method) problem characteristics to classify the literature. Although the characteristics cover most of the key elements of the LRP framework, these studies do not fully address some elements that we believe are important. Moreover, recent developments in logistics systems necessitate the alteration of some of these characteristics and the use of new dimensions of distribution logistics into the classification. 
Table 1. Problem characteristics of the classification scheme and the summary of LRP literature.

\begin{tabular}{|c|c|c|c|c|c|}
\hline 1. Hierarchical level & & 7. Number of layers & & 13. Locational decision & \\
\hline a. Delivery or pickup* & 73 & a. Two & 61 & a. At one layer & 75 \\
\hline b. Delivery and pickup & 5 & b. Three/Four* & 17 & b. At two layers* & 3 \\
\hline 2. Nature of demand & & 8. Planning period & & 14. Product & \\
\hline a. Deterministic* & 61 & a. Single* & 72 & a. Single & 74 \\
\hline b. Stochastic & 17 & b. Multiple* & 7 & b. Multiple* & 4 \\
\hline 3. Number of facilities & & 9. Time restriction & & 15. Sourcing & \\
\hline a. Single & 19 & a. No & 63 & a. Single & 77 \\
\hline b. Multiple* & 59 & b. Soft & 14 & b. Multiple* & 1 \\
\hline 4. Vehicle fleet & & c. Hard* & 1 & 16. Inventory & \\
\hline a. Single & 12 & 10. Objective function & & a. Exist & 4 \\
\hline b. Homogeneous & 52 & a. Single* & 72 & b. Not exist* & 74 \\
\hline c. Heterogeneous* & 14 & b. Multiple & 7 & 17. Solution method & \\
\hline 5. Vehicle capacity & & 11. Data & & a. Exact* & 25 \\
\hline a. Capacitated* & 47 & a. Real & 23 & b. Heuristic & 55 \\
\hline b. Uncapacitated & 31 & b. Hypothetical* & 57 & & \\
\hline 6. Facility capacity & & 12. Solution space & & & \\
\hline a. Capacitated* & 31 & a. Continuous & 4 & & \\
\hline b. Uncapacitated & 48 & b. Discrete* & 74 & & \\
\hline
\end{tabular}

Hence, we develop a classification scheme consisting of 17 problem characteristics (most of which are self-explanatory and will be explained in the next section), and classify 78 studies according to it. Table 1 exhibits our classification scheme and summarizes the classification of the studies. Asterisks indicate our problem's classification, which is explained in detail below. In addition, details of our classification are presented in Table 2 in chronological order.

\subsection{Comparison}

To better reflect the characteristics of our Mobile-ADS design problem, to express where it stands in the LRP literature, and to highlight how it differs from the previous studies, we further explain some specifics pertaining to it and compare its classification with that of the majority of LRP literature. The classification of our problem can be stated as follows.

(1a) We only consider delivery of ammo to combat units. (2a) All parameters are assumed to be fixed and known. Here, the most problematic issue is the daily ammo demand of combat units. Today, military services of almost all countries generally use three approaches (see, e.g., [31]) to estimate the amount of ammo expected to be consumed daily (consumption rate) in combat. In the first method, we predict the number of targets a weapon will encounter on a daily basis and multiply it by the required amount of ammo to destroy each target. In the second method, we predict the life of a weapon in combat before it is destroyed by an enemy. Then, we predict the number of engagements in its lifetime and multiply it by the expected ammo expenditure per engagement. In the third method, we use mathematical programming models. We define a combat scenario consisting of friendly and enemy weapons. We input several weapon and target characteristics, such as probability of hit, probability of kill, etc. Then the model gives the amount of ammo expended by each friendly weapon to defeat allocated enemy weapons. Majority of the parameters used in these three methods (expected target number, expected ammo expenditure per engagement, etc.) are based on historical data (see, e.g., [37]) and actual field experiments or tests. However, they are constantly adjusted as new data is collected. In addition, to make the predictions more accurate, these parameters change depending on the type of mission (offense, defense, etc.), terrain (desert, forest, etc.), day of the combat (first day, second day, etc.), and anticipated operational tempo. Although there is no visible way to predict daily requirements exactly, ahead of time, we are not totally in the dark. We consider anticipated daily consumption rates, calculated as explained above, and treat them as fixed demands for the sake of our model's tractability.

(3b) We locate multiple transfer points. (4c) We have two different groups of vehicles, namely, commercial and ammo trucks. In addition, in each group, we have various types of trucks that have different capacities, which in turn have different acquisition and operation costs. For example, there exist 20-, 30-, and 40-ton commercial trucks and 5-, 8-, and 10-ton ammo trucks. (5a) All trucks are capacitated. (6a) Because of manpower, terrain, enemy threat, and fire safety considerations, all transfer points have capacities. (7b) Three layers exist, and Fixed-TPs, Mobile-TPs, and combat units are located at the first, second, and third layers, respectively.

(8ab) We present both the single and multi planning period versions of our problem. Consistent with the literature and in light of our real-life application requirements, our main focus 
Table 2. Classification of the literature.

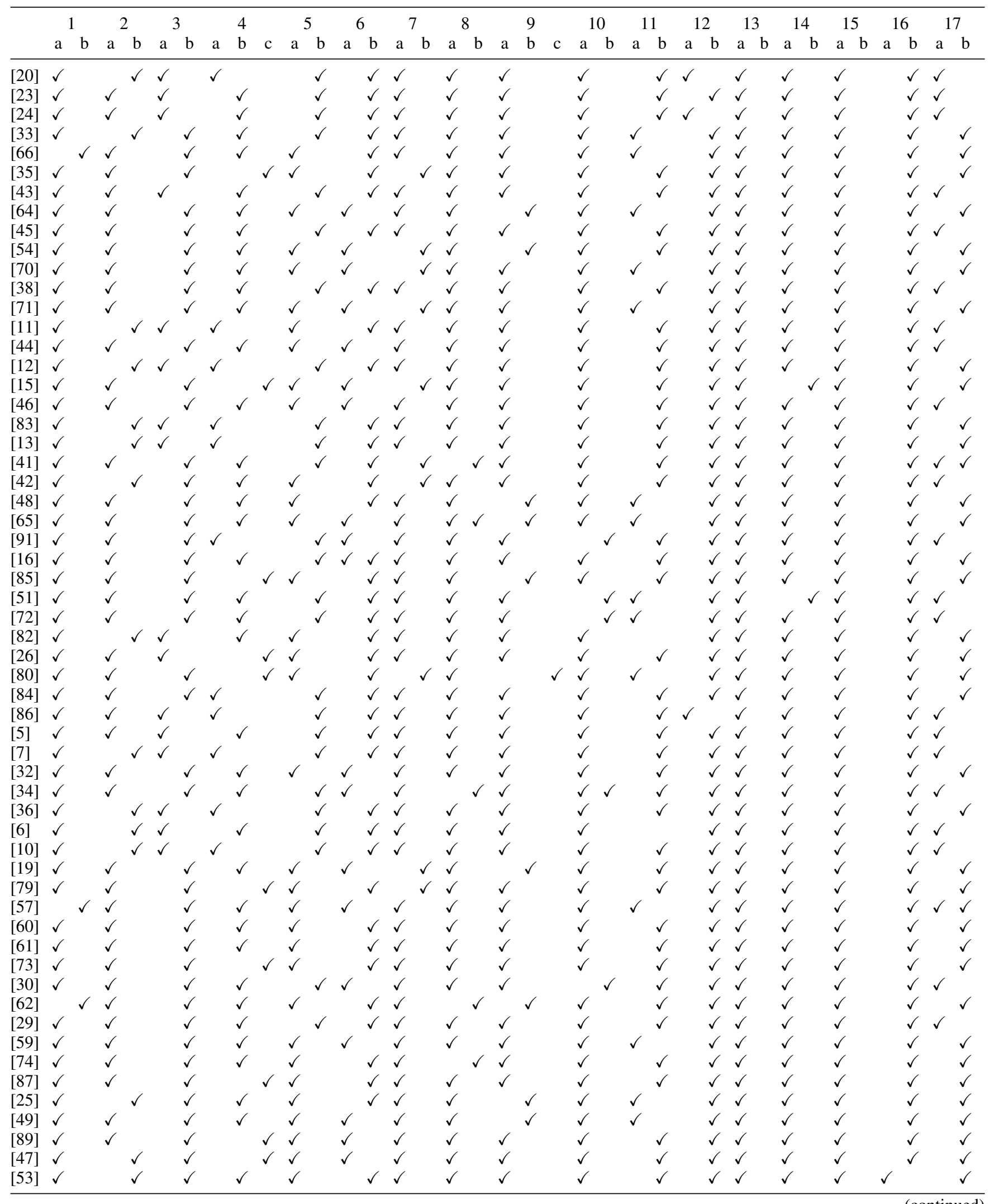


Table 2. (continued)

\begin{tabular}{|c|c|c|c|c|c|c|c|c|c|c|c|c|c|c|c|c|c|c|c|c|c|c|c|c|c|c|c|c|c|c|c|c|c|c|c|c|}
\hline & \multicolumn{2}{|c|}{1} & \multicolumn{2}{|c|}{2} & \multicolumn{2}{|c|}{3} & \multicolumn{3}{|c|}{4} & \multicolumn{2}{|c|}{5} & \multicolumn{2}{|c|}{6} & \multicolumn{2}{|c|}{7} & \multicolumn{2}{|c|}{8} & \multicolumn{3}{|c|}{9} & \multicolumn{2}{|c|}{10} & \multicolumn{2}{|c|}{11} & \multicolumn{2}{|c|}{12} & \multicolumn{2}{|c|}{13} & \multicolumn{2}{|c|}{14} & \multicolumn{2}{|c|}{15} & \multicolumn{2}{|c|}{16} & \multicolumn{2}{|c|}{17} \\
\hline & $\mathrm{a}$ & $\mathrm{b}$ & $\mathrm{a}$ & $\mathrm{b}$ & a & $\mathrm{b}$ & $\mathrm{a}$ & $\mathrm{b}$ & c & a & $\mathrm{b}$ & $\mathrm{a}$ & $\mathrm{b}$ & $\mathrm{a}$ & $\mathrm{b}$ & $\mathrm{a}$ & $\mathrm{b}$ & $\mathrm{a}$ & b & $\mathrm{c}$ & $\mathrm{a}$ & $\mathrm{b}$ & $\mathrm{a}$ & b & $\mathrm{a}$ & $\mathrm{b}$ & $\mathrm{a}$ & $\mathrm{b}$ & $\mathrm{a}$ & $\mathrm{b}$ & $\mathrm{a}$ & $\mathrm{b}$ & $\mathrm{a}$ & $\mathrm{b}$ & $\mathrm{a}$ & $\mathrm{b}$ \\
\hline [22] & $\checkmark$ & & $\checkmark$ & & & $\checkmark$ & & $\checkmark$ & & & $\checkmark$ & $\checkmark$ & & $\checkmark$ & & $\checkmark$ & & $\checkmark$ & & & $\checkmark$ & & & $\checkmark$ & & $\checkmark$ & $\checkmark$ & & $\checkmark$ & & $\checkmark$ & & & $\checkmark$ & & $\checkmark$ \\
\hline [88] & & $\checkmark$ & $\checkmark$ & & & $\checkmark$ & & & $\checkmark$ & $\checkmark$ & & & $\checkmark$ & & $\checkmark$ & $\checkmark$ & & & $\checkmark$ & & $\checkmark$ & & $\checkmark$ & & & $\checkmark$ & & $\checkmark$ & $\checkmark$ & & $\checkmark$ & & & $\checkmark$ & & $\checkmark$ \\
\hline [1] & $\checkmark$ & & $\checkmark$ & & & $\checkmark$ & & $\checkmark$ & & $\checkmark$ & & & $\checkmark$ & & $\checkmark$ & $\checkmark$ & & & $\checkmark$ & & $\checkmark$ & & & $\checkmark$ & & $\checkmark$ & $\checkmark$ & & $\checkmark$ & & $\checkmark$ & & & $\checkmark$ & & $\checkmark$ \\
\hline [4] & $\checkmark$ & & $\checkmark$ & & & $\checkmark$ & & & $\checkmark$ & $\checkmark$ & & $\checkmark$ & & & $\checkmark$ & & $\checkmark$ & $\checkmark$ & & & $\checkmark$ & & & $\checkmark$ & & $\checkmark$ & & $\checkmark$ & $\checkmark$ & & $\checkmark$ & & $\checkmark$ & & $\checkmark$ & \\
\hline [14] & $\checkmark$ & & $\checkmark$ & & & $\checkmark$ & & $\checkmark$ & & $\checkmark$ & & $\checkmark$ & & $\checkmark$ & & $\checkmark$ & & & $\checkmark$ & & $\checkmark$ & & $\checkmark$ & & & $\checkmark$ & $\checkmark$ & & $\checkmark$ & & $\checkmark$ & & & $\checkmark$ & & $\checkmark$ \\
\hline [39] & $\checkmark$ & & $\checkmark$ & & $\checkmark$ & & $\checkmark$ & & & & $\checkmark$ & & $\checkmark$ & $\checkmark$ & & $\checkmark$ & & $\checkmark$ & & & $\checkmark$ & & $\checkmark$ & $\checkmark$ & & $\checkmark$ & $\checkmark$ & & $\checkmark$ & & $\checkmark$ & & & $\checkmark$ & & $\checkmark$ \\
\hline [55] & $\checkmark$ & & $\checkmark$ & & & $\checkmark$ & & $\checkmark$ & & $\checkmark$ & & $\checkmark$ & & $\checkmark$ & & $\checkmark$ & & $\checkmark$ & & & $\checkmark$ & & & $\checkmark$ & & $\checkmark$ & $\checkmark$ & & $\checkmark$ & & $\checkmark$ & & & $\checkmark$ & & $\checkmark$ \\
\hline [76] & $\checkmark$ & & $\checkmark$ & & & $\checkmark$ & & $\checkmark$ & & & $\checkmark$ & $\checkmark$ & & $\checkmark$ & & $\checkmark$ & & $\checkmark$ & & & $\checkmark$ & & & $\checkmark$ & & $\checkmark$ & $\checkmark$ & & $\checkmark$ & & $\checkmark$ & & & $\checkmark$ & & $\checkmark$ \\
\hline [78] & $\checkmark$ & & $\checkmark$ & & $\checkmark$ & & & $\checkmark$ & & $\checkmark$ & & & $\checkmark$ & $\checkmark$ & & $\checkmark$ & & $\checkmark$ & & & $\checkmark$ & & $\checkmark$ & & $\checkmark$ & & $\checkmark$ & & $\checkmark$ & & $\checkmark$ & & & $\checkmark$ & & $\checkmark$ \\
\hline [50] & $\checkmark$ & & $\checkmark$ & & & $\checkmark$ & & $\checkmark$ & & $\checkmark$ & & $\checkmark$ & & $\checkmark$ & & $\checkmark$ & & & $\checkmark$ & & $\checkmark$ & & $\checkmark$ & $\checkmark$ & & $\checkmark$ & $\checkmark$ & & $\checkmark$ & & $\checkmark$ & & & $\checkmark$ & & $\checkmark$ \\
\hline [2] & $\checkmark$ & & $\checkmark$ & & & $\checkmark$ & & $\checkmark$ & & & $\checkmark$ & $\checkmark$ & & & $\checkmark$ & $\checkmark$ & & $\checkmark$ & & & & $\checkmark$ & $\checkmark$ & & & $\checkmark$ & & $\checkmark$ & & $\checkmark$ & $\checkmark$ & & & $\checkmark$ & $\checkmark$ & \\
\hline [8] & $\checkmark$ & & $\checkmark$ & & & $\checkmark$ & & $\checkmark$ & & $\checkmark$ & & $\checkmark$ & & $\checkmark$ & & $\checkmark$ & & $\checkmark$ & & & $\checkmark$ & & $\checkmark$ & $\checkmark$ & & $\checkmark$ & $\checkmark$ & & $\checkmark$ & & $\checkmark$ & & & $\checkmark$ & & $\checkmark$ \\
\hline [21] & $\checkmark$ & & $\checkmark$ & & & $\checkmark$ & & $\checkmark$ & & $\checkmark$ & & & $\checkmark$ & $\checkmark$ & & $\checkmark$ & & & $\checkmark$ & & & $\checkmark$ & $\checkmark$ & & & $\checkmark$ & $\checkmark$ & & $\checkmark$ & & $\checkmark$ & & & $\checkmark$ & & $\checkmark$ \\
\hline [67] & $\checkmark$ & & $\checkmark$ & & & $\checkmark$ & & $\checkmark$ & & $\checkmark$ & & & $\checkmark$ & $\checkmark$ & & $\checkmark$ & & $\checkmark$ & & & $\checkmark$ & & & $\checkmark$ & & $\checkmark$ & $\checkmark$ & & $\checkmark$ & & $\checkmark$ & & $\checkmark$ & & & $\checkmark$ \\
\hline [77] & $\checkmark$ & & & $\checkmark$ & & $\checkmark$ & & $\checkmark$ & & & $\checkmark$ & $\checkmark$ & & $\checkmark$ & & $\checkmark$ & & $\checkmark$ & & & $\checkmark$ & & & $\checkmark$ & & $\checkmark$ & $\checkmark$ & & $\checkmark$ & & $\checkmark$ & & & $\checkmark$ & & $\checkmark$ \\
\hline [81] & $\checkmark$ & & & $\checkmark$ & & $\checkmark$ & & $\checkmark$ & & $\checkmark$ & & & $\checkmark$ & & $\checkmark$ & $\checkmark$ & & $\checkmark$ & & & $\checkmark$ & & & $\checkmark$ & & $\checkmark$ & $\checkmark$ & & $\checkmark$ & & $\checkmark$ & & $\checkmark$ & & & $\checkmark$ \\
\hline [90] & & $\checkmark$ & $\checkmark$ & & & $\checkmark$ & & & $\checkmark$ & $\checkmark$ & & $\checkmark$ & & & $\checkmark$ & & $\checkmark$ & $\checkmark$ & & & $\checkmark$ & & & $\checkmark$ & & $\checkmark$ & $\checkmark$ & & & $\checkmark$ & & $\checkmark$ & & $\checkmark$ & & $\checkmark$ \\
\hline [9] & $\checkmark$ & & $\checkmark$ & & $\checkmark$ & & & $\checkmark$ & & & $\checkmark$ & & $\checkmark$ & $\checkmark$ & & $\checkmark$ & & $\checkmark$ & & & $\checkmark$ & & & $\checkmark$ & & $\checkmark$ & $\checkmark$ & & $\checkmark$ & & $\checkmark$ & & & $\checkmark$ & & $\checkmark$ \\
\hline [27] & $\checkmark$ & & $\checkmark$ & & & $\checkmark$ & & $\checkmark$ & & $\checkmark$ & & $\checkmark$ & & $\checkmark$ & & $\checkmark$ & & $\checkmark$ & & & $\checkmark$ & & & $\checkmark$ & & $\checkmark$ & $\checkmark$ & & $\checkmark$ & & $\checkmark$ & & & $\checkmark$ & & $\checkmark$ \\
\hline [3] & $\checkmark$ & & $\checkmark$ & & & $\checkmark$ & & & $\checkmark$ & $\checkmark$ & & $\checkmark$ & & & $\checkmark$ & $\checkmark$ & & $\checkmark$ & & & $\checkmark$ & & $\checkmark$ & $\checkmark$ & & $\checkmark$ & $\checkmark$ & & $\checkmark$ & & $\checkmark$ & & & $\checkmark$ & & $\checkmark$ \\
\hline
\end{tabular}

is the static version, for which we can suggest a computationally more viable model. The dynamic model, which is valid for at least two planning periods, is only presented following the discussion of the static model. Overall, we consider consecutive 24-h planning periods, since each combat unit possesses a specific amount of ammo on hand to initiate and continue combat operations for $24 \mathrm{~h}$, until it is supplied from the rear.

(9c) The battlefield is open to unexpected circumstances. At different times of combat, depending on the combat type and enemy threat, some ammo types may become more valuable, and combat units may require them more urgently than other types of ammo. Therefore, there are different time deadlines for each type of ammo and for each combat unit. In addition, supplying a unit with ammo requires the unit to halt for some time and take the required precautions, such as perimeter security. A combat unit cannot always halt in battle, especially when it is actively engaged with an enemy. Hence, after combat starts, units need some time to gain positions that render them available for supply, and this time constitutes the earliest time that a unit can receive its supplies. In summary, we have two-sided time windows, i.e., hard time restrictions, in our problem.

(10a) We have a single objective function (explained in more detail below) that unifies multiple cost components. (11b) Since this is a military application on a sensitive topic, all data we present in this article is hypothetical. (12b) The operation plan of a battle dictates the targets of each combat unit and the time by which each target is supposed to be seized. In other words, this plan includes the daily anticipated locations of each unit, and we take these locations as given. In addition, transfer points cannot be located just anywhere on the battlefield. Such a site should possess characteristics to allow logistics support operations as well as a tactical defense against enemy threats. Logistics planners consider these characteristics, and perform on-site or map reconnaissance to determine potential locations before battle commences. Therefore, transfer points can only be located at these predefined potential locations, and, hence, our solution space is discrete. (13b) We locate Fixed-TPs and MobileTPs at the first and second layers, respectively; locational decisions exist at two different layers. (14b) On the battlefield, combat units need and use several types of ammo (for example, rifle bullets, artillery munition, and hand grenades). They need them at different locations, at different times, and at different rates. Hence, we need to determine the schedule of each ammo type separately, which makes our problem a multi-product problem. (15b) In an LRP with single sourcing, each customer is to be supplied by exactly one vehicle or depot. In our problem, even the same ammo type may be brought to a unit by two or more different trucks. We, therefore, modeled for multi sourcing. (16b) We do not hold inventory at transfer points. (17b) We solve the model exactly with a commercial solver.

In summary, we utilize a capacitated heterogeneous vehicle fleet, whereas the literature generally use a capacitated homogeneous fleet. Furthermore, the majority of the literature considers uncapacitated facilities, but we consider capacitated ones. Most of the literature studies two layers, whereas our model has three layers. Except for seven studies, the 


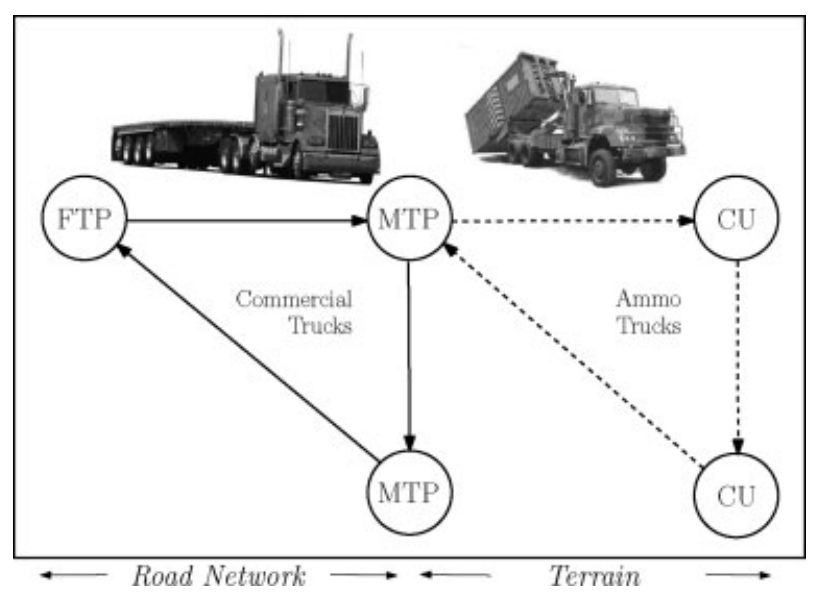

Figure 2. Routes of commercial and ammo trucks.

literature concentrates on static LRP problems. Although we primarily focus on the static version, we additionally provide an extension to the multi-period dynamic case. The time restriction issue is rarely incorporated within the context of the literature. Only one study includes hard time restrictions as we do. Locational decisions exist at only one layer in all studies except for three. However, we locate facilities at two different layers. There are only four studies that distribute multiple products as we do. Last of all, although the literature mostly involves single sourcing (a customer is allowed to be served by at most two vehicles in the model of [62], but one for pickups and one for deliveries), we utilize multiple sourcing.

This brief analysis illustrates that some characteristics of our problem are rarely included in the previous models. This article is directly aimed at handling these aspects and incorporating them into a computationally viable mathematical model. To the best of the authors' knowledge, even the static version of our study is the first attempt to construct such an inclusive real world LRP model.

\section{STATIC MODEL DEVELOPMENT AND VALID INEQUALITIES}

In this section, we present the mathematical formulation of the Mobile-ADS design problem for a fixed period and derive several valid inequalities to speed up the solution time. We promote this model as a decision support tool during the short-term tactical decision making process. Section 4 displays the computational viability of the static model improved with valid inequalities in several realistically sized scenarios. Section 5 extends the static model to the case in which we have at least two periods within the planning horizon. Because of its computational burden, the dynamic model is reserved for long-term strategic planning.
We model the battlefield as a network of three types of nodes, i.e., potential Fixed-TP and Mobile-TP and known combat unit locations. With this representation, we consider Mobile-ADS, shown in Figure 1, as a directed and connected network $G=(N, A)$ that is defined by a set $N$ of nodes and a set $A$ of arcs. Arc set $A$ consists of two types of mutually exclusive road networks as shown in Fig. 2. The first is the two-way road network, on which commercial trucks travel among Fixed-TPs and Mobile-TPs. The second is the two-way trace network on the battle terrain, on which ammo trucks travel among Mobile-TPs and combat units. Model specifications used in the formulation are listed in Table 3.

Table 3. Model specifications.

Sets

$N$ : $\quad$ Set of all nodes such that $N=N_{\mathrm{F}} \cup N_{\mathrm{M}} \bigcup N_{\mathrm{C}}$ and $N_{\mathrm{F}}$, $N_{\mathrm{M}}, N_{\mathrm{C}}$ are mutually exclusive.

$N_{\mathrm{F}}$ : $\quad$ Set of potential Fixed-TP nodes such that $N_{\mathrm{F}} \subset N$.

$N_{\mathrm{M}}$ : $\quad$ Set of potential Mobile-TP nodes such that $N_{\mathrm{M}} \subset N$.

$N_{\mathrm{C}}: \quad$ Set of combat unit nodes such that $N_{\mathrm{C}} \subset N$.

Note that $N_{\mathrm{FM}}=N_{\mathrm{F}} \bigcup N_{\mathrm{M}}$ and $N_{\mathrm{MC}}=N_{\mathrm{M}} \bigcup N_{\mathrm{C}}$.

$V: \quad$ Set of all vehicles such that $V=V_{\mathrm{F}} \bigcup V_{\mathrm{M}}$ and $V_{\mathrm{F}}, V_{\mathrm{M}}$ are mutually exclusive.

$V_{\mathrm{F}}: \quad$ Set of commercial trucks (all stationed at Fixed-TPs)

such that $V_{\mathrm{F}} \subset V$.

$V_{\mathrm{M}}: \quad$ Set of ammo trucks (all stationed at Mobile-TPs) such

that $V_{\mathrm{M}} \subset V$.

$P$ : $\quad$ Set of ammo types.

Parameters

$Q_{i p}: \quad$ Demand of combat unit $i$ for ammo type $p$.

$\mathrm{CD}_{i p}$ : Non-negative capacity of transfer point $i$ for ammo type

$\mathrm{CV}_{\mathrm{vp}}$ : Non-negative capacity of vehicle $\mathrm{v}$ for ammo type $p$.

$\mathrm{CT}_{\mathrm{v}}$ : $\quad$ Non-negative total capacity of vehicle $\mathrm{v}$.

$\mathrm{TI}_{i j}$ : $\quad$ Travel time between nodes $i$ and $j$, which includes the service time at nodes.

$\mathrm{TE}_{i p}$ : $\quad$ Earliest time that combat unit $i$ can receive supplies of ammo type $p$.

$\mathrm{TL}_{i p}$ : Latest time that combat unit $i$ can receive supplies of ammo type $p$.

$\mathrm{TM}_{p}$ : $\quad$ Maximum latest arrival time of ammo type $p$ among units, that is $\mathrm{TM}_{p}=\max _{i \in N_{\mathrm{C}}}\left\{\mathrm{TL}_{i p}\right\}$.

TM : $\quad$ Maximum of the latest arrival times of all ammo types, that is $\mathrm{TM}=\max _{p \in P}\left\{\mathrm{TM}_{p}\right\}$.

$\mathrm{TC}_{\mathrm{vp}}$ : Cost of transporting one unit of ammo type $p$ on vehicle v per hour.

$\mathrm{VC}_{\mathrm{v}}$ : Cost of acquiring vehicle $\mathrm{v}$.

$\mathrm{DC}_{\mathrm{v}}$ : Cost of driving vehicle $\mathrm{v}$ per hour.

$\mathrm{FC}_{i}$ : $\quad$ Fixed cost of opening transfer point $i$.

Decision variables

$f_{i j \mathrm{v} p}$ : $\quad$ Non-negative amount of flow of ammo type $p$ carried from node $i$ to $j$ by vehicle $\mathrm{v}$.

$\operatorname{tp}_{i p}$ : $\quad$ Non-negative arrival time of ammo type $p$ at node $i$.

$\mathrm{tv}_{i \mathrm{v}}$ : $\quad$ Non-negative arrival time of vehicle $\mathrm{v}$ at node $i$.

$y_{i}$ : $\quad 1$, if transfer point $i$ is opened; 0 otherwise.

$x_{i j \mathrm{v}}: \quad 1$, if vehicle v travels from node $i$ to $j ; 0$ otherwise.

$w_{i j p}: \quad 1$, if ammo type $p$ travels from node $i$ to $j ; 0$ otherwise. 


\subsection{Constraints}

We enumerate separate constraints as usual, such as 1,2 , etc. If we write the same constraint several times for disjoint index sets, then we enumerate them, such as $1 \mathrm{a}, 1 \mathrm{~b}, \ldots$ or $2 \mathrm{a}$, $2 \mathrm{~b}, \ldots$ and so on.

\subsubsection{Product Flow Balance Constraints}

Constraints (1) ensure that inflow to a Mobile-TP or combat unit is equal to the sum of the total outflow from that node and the demand of that node:

$$
\begin{gathered}
\sum_{\mathrm{v} \in V_{\mathrm{F}}}\left(\sum_{\substack{j \in N_{\mathrm{F}} \\
j \neq i}} f_{j i v p}-\sum_{\substack{j \in N_{\mathrm{M}} \\
j \neq i}} f_{i j v p}\right) \\
=\sum_{\mathrm{v} \in V_{\mathrm{M}}} \sum_{\substack{j \in N_{\mathrm{C}} \\
f_{i j v p}}} \forall i \in N_{\mathrm{M}}, p \in P \\
\sum_{\mathrm{v} \in V_{\mathrm{M}}}\left(\sum_{\substack{j \in N_{\mathrm{MC}} \\
j \neq i}} f_{j i v p}-\sum_{\substack{j \in N_{\mathrm{C}} \\
j \neq i}} f_{i j v p}\right)=Q_{i p} \quad \forall i \in N_{\mathrm{C}}, p \in P
\end{gathered}
$$

Note that constraints (1b) declare the problem infeasible if the total demand cannot be satisfied for some reason, such as a lack of ammo or trucks or transfer points or tight time windows. In such situations, instead of giving no solution, we may want to provide the best solution that can be obtained with available resources. To do so, we need to allow the demand satisfaction limitation to be overruled at a certain cost by rewriting the hard constraints (1b) as soft constraints, as follows $\sum_{\mathrm{v} \in V_{\mathrm{M}}}\left(\sum_{\substack{j \in N_{\mathrm{MC}} \\ j \neq i}} f_{j i v p}-\sum_{\substack{j \in N_{\mathrm{C}} \\ j \neq i}} f_{i j v p}\right)+u_{i p}=Q_{i p}$ where $u_{i p}$ is a non-negative decision variable, indicating the amount of unmet demand of ammo type $p$ at combat unit $i$, with a high enough positive cost coefficient in the objective function.

Constraints (2) guarantee that a vehicle cannot leave a node with a heavier load than it was carrying before entering into that node:

$$
\begin{aligned}
& \sum_{\substack{j \in N_{\mathrm{FM}} \\
j \neq i}} f_{j i v p} \geq \sum_{\substack{j \in N_{\mathrm{M}} \\
j \neq i}} f_{i j v p} \quad \forall i \in N_{\mathrm{M}}, \mathrm{v} \in V_{\mathrm{F}}, p \in P \\
& \sum_{\substack{j \in N_{\mathrm{MC}} \\
j \neq i}} f_{j i v p} \geq \sum_{\substack{j \in N_{\mathrm{C}} \\
j \neq i}} f_{i j v p} \quad \forall i \in N_{\mathrm{C}}, \mathrm{v} \in V_{\mathrm{M}}, p \in P
\end{aligned}
$$

\subsubsection{Vehicle Flow Balance Constraints}

Constraints (3) maintain that a vehicle can start its route from one and only one transfer point. Constraints (4) force each vehicle to turn back to its home transfer point, where it is allocated. Constraints (5) require that each vehicle leave the node that it enters:

$$
\begin{aligned}
& \sum_{i \in N_{\mathrm{F}}} \sum_{j \in N_{\mathrm{M}}} x_{i j v} \leq 1 \quad \forall \mathrm{v} \in V_{\mathrm{F}} \\
& \sum_{i \in N_{\mathrm{M}}} \sum_{j \in N_{\mathrm{C}}} x_{i j v} \leq 1 \quad \forall \mathrm{v} \in V_{\mathrm{M}} \\
& \sum_{j \in N_{\mathrm{M}}} x_{j i v}=\sum_{j \in N_{\mathrm{M}}} x_{i j v} \quad \forall i \in N_{\mathrm{F}}, \mathrm{v} \in V_{\mathrm{F}} \\
& \sum_{j \in N_{\mathrm{C}}} x_{j i v}=\sum_{j \in N_{\mathrm{C}}} x_{i j v} \quad \forall i \in N_{\mathrm{M}}, \mathrm{v} \in V_{\mathrm{M}} \\
& \sum_{\substack{j \in N_{\mathrm{FM}} \\
j \neq i}} x_{j i v}=\sum_{\substack{j \in N_{\mathrm{FM}} \\
j \neq i}} x_{i j v} \quad \forall i \in N_{\mathrm{M}}, \mathrm{v} \in V_{\mathrm{F}} \\
& \sum_{\substack{j \in N_{\mathrm{MC}} \\
j \neq i}} x_{j i v}=\sum_{\substack{j \in N_{\mathrm{MC}} \\
j \neq i}} x_{i j v} \quad \forall i \in N_{\mathrm{C}}, \mathrm{v} \in V_{\mathrm{M}}
\end{aligned}
$$

\subsubsection{Capacity Constraints}

Constraints (6) ensure that transfer points cannot send more than their capacity for that ammo type. They also guarantee that there is no flow from or through any closed transfer point. Constraints (7) require that vehicle capacities are not exceeded. All vehicles also have total capacities that are respected by constraints (8):

$$
\begin{aligned}
& \sum_{\mathrm{v} \in V_{\mathrm{F}}} \sum_{j \in N_{\mathrm{M}}} f_{i j v p} \leq \mathrm{CD}_{i p} \cdot y_{i} \quad \forall i \in N_{\mathrm{F}}, p \in P \\
& \sum_{\mathrm{v} \in V_{\mathrm{M}}} \sum_{j \in N_{\mathrm{C}}} f_{i j v p} \leq \mathrm{CD}_{i p} \cdot y_{i} \quad \forall i \in N_{\mathrm{M}}, p \in P \\
& \sum_{\mathrm{v} \in V_{\mathrm{F}}} \sum_{\substack{j \in N_{\mathrm{M}} \\
j \neq i}} f_{i j v p} \leq\left(\sum_{l \in N_{\mathrm{C}}} Q_{l p}\right) \cdot y_{i} \quad \forall i \in N_{\mathrm{M}}, p \in P \\
& f_{i j v p} \leq \mathrm{CV}_{\mathrm{vp}} \cdot x_{i j v} \forall i \in N_{\mathrm{F}}, j \in N_{\mathrm{M}}, \mathrm{v} \in V_{\mathrm{F}}, p \in P ; \\
& \forall i, j \in N_{\mathrm{M}}, i \neq j, \mathrm{v} \in V_{\mathrm{F}}, p \in P ; \\
& \forall i \in N_{\mathrm{M}}, j \in N_{\mathrm{C}}, \mathrm{v} \in V_{\mathrm{M}}, p \in P ; \\
& \forall i, j \in N_{\mathrm{C}}, i \neq j, \mathrm{v} \in V_{\mathrm{M}}, p \in P
\end{aligned}
$$

$$
\begin{gathered}
\sum_{p \in P} f_{i j v p} \leq \mathrm{CT}_{\mathrm{v}} \cdot x_{i j v} \quad \forall i \in N_{\mathrm{F}}, j \in N_{\mathrm{M}}, \mathrm{v} \in V_{\mathrm{F}} ; \\
\forall i, j \in N_{\mathrm{M}}, i \neq j, \mathrm{v} \in V_{\mathrm{F}} \\
\forall i \in N_{\mathrm{M}}, j \in N_{\mathrm{C}}, \mathrm{v} \in V_{\mathrm{M}} ; \forall i, j \in N_{\mathrm{C}}, i \neq j, \mathrm{v} \in V_{\mathrm{M}}
\end{gathered}
$$




\subsubsection{Relation Constraints}

Constraints (9) require that a truck carry some amount of ammo if it is dispatched. Constraints (10) and (11) set the correct logical relationships between the decision variables $f$ and $w$ :

$\sum_{p \in P} f_{i j v p} \geq x_{i j v}$

$\forall i \in N_{\mathrm{F}}, j \in N_{\mathrm{M}}, \mathrm{v} \in V_{\mathrm{F}} ; \forall i, j \in N_{\mathrm{M}}, i \neq j, \mathrm{v} \in V_{\mathrm{F}}$

$\forall i \in N_{\mathrm{M}}, j \in N_{\mathrm{C}}, \mathrm{v} \in V_{\mathrm{M}} ; \forall i, j \in N_{\mathrm{C}}, i \neq j, \mathrm{v} \in V_{\mathrm{M}}$

$$
\begin{gathered}
\left(\sum_{l \in N_{\mathrm{C}}} Q_{l p}\right) \cdot w_{i j p} \geq f_{i j v p} \forall i \in N_{\mathrm{F}}, j \in N_{\mathrm{M}}, \mathrm{v} \in V_{\mathrm{F}}, p \in P \\
\quad \forall i, j \in N_{\mathrm{M}}, i \neq j, \mathrm{v} \in V_{\mathrm{F}}, p \in P \\
\quad \forall i \in N_{\mathrm{M}}, j \in N_{\mathrm{C}}, \mathrm{v} \in V_{\mathrm{M}}, p \in P \\
\quad \forall i, j \in N_{\mathrm{C}}, i \neq j, \mathrm{v} \in V_{\mathrm{M}}, p \in P
\end{gathered}
$$

$\sum_{\mathrm{v} \in V_{\mathrm{F}}} f_{i j v p} \geq w_{i j p}$

$\forall i \in N_{\mathrm{F}}, j \in N_{\mathrm{M}}, p \in P ; \forall i, j \in N_{\mathrm{M}}, i \neq j, p \in P$

$\sum_{\mathbf{v} \in V_{\mathrm{M}}} f_{i j v p} \geq w_{i j p}$

$\forall i \in N_{\mathrm{M}}, j \in N_{\mathrm{C}}, p \in P ; \forall i, j \in N_{\mathrm{C}}, i \neq j, p \in P$

Note that in constraints (9) and (11), ammo flow is measured in undefined units. Hence, one needs to be careful about defining the unit of flow, because these constraints do not permit a truck to carry an ammo type of less than 1 unit. If one wants to do so, then the right hand sides should be multiplied by an appropriate multiplier. For example, if our unit is 1 ton, and if we do not want to carry an ammo type less than 0.2 tons with a single truck, then our multiplier would be 0.2 .

\subsubsection{Time Related Constraints}

Constraints (12) impose the time window requirements of combat units for all ammo types. Constraints (13) define the initial condition of arrival times. Constraints (14) compute the arrival times of ammo types at nodes:

$$
\begin{aligned}
\mathrm{TE}_{i p} \leq \mathrm{tp}_{i p} \leq \mathrm{TL}_{i p} & \forall i \in N_{\mathrm{C}}, p \in P \\
\operatorname{tp}_{i p}=0 & \forall i \in N_{\mathrm{F}}, p \in P
\end{aligned}
$$

$$
\begin{aligned}
& \operatorname{tp}_{i p}+\mathrm{TI}_{i j} \cdot w_{i j p}-\mathrm{TM}_{p} \cdot\left(1-w_{i j p}\right) \leq \mathrm{tp}_{j p} \\
& \forall i \in N_{\mathrm{F}}, j \in N_{\mathrm{M}}, p \in P ; \forall i, j \in N_{\mathrm{M}}, i \neq j, p \in P \\
& \forall i \in N_{\mathrm{M}}, j \in N_{\mathrm{C}}, p \in P ; \forall i, j \in N_{\mathrm{C}}, i \neq j, p \in P
\end{aligned}
$$

Constraints (12)-(14) ensure that the latest ammo arrivals respect the time windows of units. Note that waiting for ammo at combat units is allowed, and, in the context of this article, a time window indicates the time interval in which a unit can halt in battle and receive the waiting or newly arrived supplies. Hence, ammo is allowed to reach a unit before the earliest time and wait there until the unit actually takes it.

Recall that $w_{i j p}$ does not carry any information about vehicles. Hence, constraints (12)-(14) cannot prevent sub-tours of vehicles. To remedy this condition, we introduce the subtour elimination constraints of [56] as constraints (15):

$$
\begin{aligned}
\operatorname{tv}_{i v}+ & \mathrm{TI}_{i j} \cdot x_{i j v}-\mathrm{TM} \cdot\left(1-x_{i j v}\right) \leq \mathrm{tv}_{j v} \\
& \forall i \in N_{\mathrm{F}}, j \in N_{\mathrm{M}}, \mathrm{v} \in V_{\mathrm{F}} ; \forall i, j \in N_{\mathrm{M}}, i \neq j, \mathrm{v} \in V_{\mathrm{F}} \\
& \forall i \in N_{\mathrm{M}}, j \in N_{\mathrm{C}}, \mathrm{v} \in V_{\mathrm{M}} ; \forall i, j \in N_{\mathrm{C}}, i \neq j, \mathrm{v} \in V_{\mathrm{M}}
\end{aligned}
$$

\subsection{Objective Function}

In the Mobile-ADS design problem, two different objectives exist of which each could be applicable depending on the situation. The first objective considers the costs of transfer point establishment, vehicle acquisition, and ammo distribution. The second one considers again the costs of transfer point establishment and vehicle acquisition plus the cost of truck driving. As can be seen, two of the cost components are common to both objectives and are shown below:

$$
\begin{aligned}
& \sum_{i \in N_{\mathrm{FM}}} \mathrm{FC}_{i} \cdot y_{i} \\
& \sum_{i \in N_{\mathrm{F}}} \sum_{j \in N_{\mathrm{M}}} \sum_{\mathrm{v} \in V_{\mathrm{F}}} \mathrm{VC}_{\mathrm{v}} \cdot x_{i j v}+\sum_{i \in N_{\mathrm{M}}} \sum_{j \in N_{\mathrm{C}}} \sum_{\mathrm{v} \in V_{\mathrm{M}}} \mathrm{VC}_{\mathrm{v}} \cdot x_{i j v}
\end{aligned}
$$

Equation (16) is the total fixed cost of opening transfer points, and Eq. (17) is the total acquisition cost of used trucks. Now, we present the last component of each objective.

Depending on the mission, available forces, enemy threat, or country economy, for example, different factors may gain more importance or urgency above others on the battlefield. If we put economy and financial concerns above others, then the total transportation cost of ammo becomes critical. This cost constitutes the third component of the first objective and is shown below:

$$
\sum_{i \in N} \sum_{j \in N} \sum_{\mathrm{v} \in V} \sum_{p \in P} \mathrm{TC}_{\mathrm{vp}} \cdot \mathrm{TI}_{i j} \cdot f_{i j v p}
$$

If an enemy has the ability to detect our logistics convoys, then the more traffic we have, the more our convoys are exposed to enemy fire. Moreover, we may want to concentrate some of our forces on a particular region of the combat area without the enemy's notice. In such circumstances, stealth becomes a big concern, and we want minimal traffic on the 
battlefield. Hence, the total driving time of vehicles becomes critical, and constitutes the third component of the second objective that is shown below:

$$
\sum_{i \in N} \sum_{j \in N} \sum_{\mathrm{v} \in V} \mathrm{DC}_{\mathrm{v}} \cdot \mathrm{TI}_{i j} \cdot x_{i j v}
$$

To summarize, the first objective function is $z 1=(16)+$ $(17)+(18)$, and the second is $z 2=(16)+(17)+(19)$. It is important to note that on the same battlefield and at the same time, different objectives may gain priority for different units. For example, one brigade may move in a different direction in concealment, whereas others keep their positions as they are. Hence, for the first brigade $z 2$ and for the rest $z 1$ becomes the objective on the same battlefield at the same time.

\subsection{Valid Inequalities}

In this section, we present several valid inequalities (VIs) to reduce the solution time. For the sake of brevity, when the same VI is needed several times between Fixed-TPs and Mobile-TPs, among Mobile-TPs, between Mobile-TPs and combat units and among combat units, we only give it once (for example, only between Fixed-TPs and Mobile-TPs). It is clear that they must be duplicated for the other layers. Our VIs are shown below:

$$
\begin{aligned}
& \sum_{\mathrm{v} \in V_{\mathrm{F}}} \sum_{i \in N_{\mathrm{F}}} \sum_{j \in N_{\mathrm{M}}} f_{i j v p}=\sum_{i \in N_{\mathrm{C}}} Q_{i p} \quad \forall p \in P \\
& \sum_{\substack{j \in N_{\mathrm{FM}} \\
j \neq i}} x_{i j v} \leq 1 \quad \forall i \in N_{\mathrm{M}}, \mathrm{v} \in V_{\mathrm{F}} \\
& \sum_{\mathrm{v} \in V_{\mathrm{F}}} \sum_{i \in N_{\mathrm{F}}} \sum_{j \in N_{\mathrm{M}}} x_{i j v} \geq\left\lceil\frac{\sum_{p \in P} \sum_{i \in N_{\mathrm{C}}} Q_{i p}}{\max _{\mathrm{v} \in V_{\mathrm{F}}}\left\{\mathrm{CT}_{\mathrm{v}}\right\}}\right] \\
& w_{i j p} \leq y_{i} \quad \forall i \in N_{\mathrm{F}}, j \in N_{\mathrm{M}}, p \in P \\
& \sum_{p \in P} \sum_{j \in N_{\mathrm{M}}} w_{i j p} \geq y_{i} \quad \forall i \in N_{\mathrm{F}} \\
& w_{i j p} \leq \sum_{\mathrm{v} \in V_{\mathrm{F}}} x_{i j v} \quad \forall i \in N_{\mathrm{F}}, j \in N_{\mathrm{M}}, p \in P \\
& \sum_{p \in P} w_{i j p} \geq x_{i j v} \quad \forall i \in N_{\mathrm{F}}, j \in N_{\mathrm{M}}, \mathrm{v} \in V_{\mathrm{F}} \\
& \sum_{j \in N_{\mathrm{M}}} x_{i j v} \leq y_{i} \quad \forall i \in N_{\mathrm{F}}, \mathrm{v} \in V_{\mathrm{F}} \\
& \sum_{\mathrm{v} \in V_{\mathrm{F}}} \sum_{j \in N_{\mathrm{M}}} x_{i j v} \geq y_{i} \quad \forall i \in N_{\mathrm{F}} \\
& \sum_{\mathrm{v} \in V_{\mathrm{F}}} \sum_{j \in N_{\mathrm{M}}} x_{i j v} \leq\left|V_{\mathrm{F}}\right| \cdot y_{i} \quad \forall i \in N_{\mathrm{F}} \\
& \left.\sum_{i \in N_{\mathrm{F}}} y_{i} \geq \mid \frac{\sum_{p \in P} \sum_{i \in N_{\mathrm{C}}} Q_{i p}}{\max _{p \in P, i \in N_{\mathrm{F}}}\left\{\mathrm{CD}_{i p}\right\}}\right]
\end{aligned}
$$

$$
\sum_{\substack{i \in N_{\mathrm{MC}} \\ i \neq j}} \sum_{j \in N_{\mathrm{C}}} \mathrm{TI}_{i j} \cdot x_{i j v} \leq \mathrm{TM}-\min _{i \in N_{\mathrm{F}}, j \in N_{\mathrm{M}}}\left\{\mathrm{TI}_{i j}\right\} \quad \forall \mathrm{v} \in V_{\mathrm{M}}
$$

VI 1 requires that outflow from transfer points be equal to the total demand of all combat units for each ammo type. VI 2 maintains that a truck cannot travel from a node to two or more nodes. VI 3 sets a lower bound for the total number of trucks that must be dispatched from transfer points. VI 4 ensures that ammo types cannot pass through closed transfer points. VI 5 provides that at least one ammo type must pass through an open transfer point. VI 6 states that if an ammo type travels from node $i$ to $j$, then there must exist at least one truck traveling between these two nodes. VI 7 maintains the reverse condition by preventing any truck from traveling between nodes $i$ and $j$ if no ammo type travels in between. VI 8 provides that no truck can be dispatched from or pass through a closed transfer point. VI 9 requires that an open transfer point dispatches at least one truck. VI 10 guarantees that an open transfer point cannot dispatch more trucks than exist in the system. VI 11 sets a lower bound for the number of opened transfer points. VI 12 sets an upper bound for the total travel time of the modified tours of ammo trucks that are obtained by deleting the returning arc of each tour.

\section{COMPUTATIONAL EXPERIMENTS}

In this section, we first test our VIs on six moderate sized test problem instances (PIs). Then, we report the performance of our model on several realistically sized scenarios. All computations are conducted on a laptop computer with $1.83 \mathrm{GHz}$ CPU, 1 GB RAM, and Windows XP [69] operating system. We use GAMS/Cplex 9.1 [28] as the solver and GAMS 22.0 [17] as the modeling language.

\subsection{Experiments on Test Bed Problem Instances}

In each PI, we have $\left|N_{\mathrm{F}}\right|=3,\left|N_{\mathrm{M}}\right|=8,\left|N_{\mathrm{C}}\right|=10$, $\left|V_{\mathrm{F}}\right|=5,\left|V_{\mathrm{M}}\right|=10$, and $|P|=3$. In PI 1, 3, and 5 (PI 2, 4, and 6) at least one Fixed-TP, and two (three) Mobile-TPs must be opened, and two (three) commercial, and three (five) ammo trucks must be dispatched to satisfy the total demand. In addition, PI 1 and 2 have three different time windows of which combat units $1,2,3(4,5,6,7$ and $8,9,10)$ respect the first (second and third) one for each type of ammo. Likewise, PI 3 and 4 have four, and PI 5 and 6 have six different time windows.

We use strong branching for selecting the branching variable and best-estimate search for selecting the next node when backtracking. For the other parameters, we use GAMS/Cplex's default settings. When comparing valid inequalities, we base the comparison on the optimality gap 
Table 4. Performance of the valid inequalities with the first objective.

\begin{tabular}{|c|c|c|c|c|c|c|c|c|c|}
\hline & \multicolumn{9}{|c|}{ Gaps (\%) after $1 \mathrm{~h}$} \\
\hline & PI 1 & PI 2 & PI 3 & PI 4 & PI 5 & PI 6 & Min & Average & $\operatorname{Max}$ \\
\hline Original & 16.36 & - & 15.76 & 4.35 & 16.80 & - & - & - & - \\
\hline VI 1 & 20.64 & 9.86 & 14.87 & 23.22 & 12.19 & 17.46 & 9.86 & 16.37 & 23.22 \\
\hline VI 2 & 14.44 & 8.45 & 11.16 & 0.61 & 16.16 & 14.11 & 0.61 & 10.82 & 16.16 \\
\hline VI 3 & 16.53 & - & 8.60 & 5.32 & 9.96 & 5.08 & - & - & - \\
\hline VI 4 & 11.96 & 7.93 & 10.41 & 4.79 & 17.69 & 6.11 & 4.79 & 9.82 & 17.69 \\
\hline VI 5 & 19.85 & 1.39 & 16.84 & 5.09 & 20.34 & 2.10 & 1.39 & 10.94 & 20.34 \\
\hline VI 6 & 32.77 & - & 16.02 & - & 23.56 & - & - & - & - \\
\hline VI 7 & 15.96 & - & 12.11 & 5.22 & 19.08 & 5.13 & - & - & - \\
\hline VI 8 & 15.39 & 5.47 & 21.37 & 8.95 & 18.06 & 4.50 & 4.50 & 12.29 & 21.37 \\
\hline VI 9 & 15.37 & 5.32 & 10.03 & 6.42 & 14.10 & - & - & - & - \\
\hline VI 10 & 16.36 & - & 15.76 & 4.35 & 16.83 & - & - & - & - \\
\hline VI 11 & 5.69 & 13.47 & 10.29 & - & 14.26 & 9.75 & - & - & - \\
\hline VI 12 & 12.38 & 1.78 & 8.60 & 7.86 & 10.74 & 4.45 & 1.78 & 7.64 & 12.38 \\
\hline
\end{tabular}

reported by GAMS/Cplex. Computations for a problem are terminated after $3600 \mathrm{~s}$.

We compare the performance of VIs with the first objective in Table 4. The results with the second objective are not given, for brevity. The first row presents the gaps without any VI, and dashes indicate that no feasible solution can be found within the given time limit. Subsequent rows demonstrate the gaps of the formulations with the corresponding VIs. Since VI 2, 4, and 12 are the best performing ones, we first test all pairwise combinations of other VIs with them. The results show that no pairwise combination is superior to them. Following the same methodology, we test all triple combinations of other VIs with some promising pairwise combinations, and no improvement is obtained. Then we test them in larger PIs (with $\left|N_{\mathrm{C}}\right|=20$ ) and finally conclude that VI 2 improves the performance of our model better than the others.

\subsection{Experiments on Realistically Sized Problem Instances}

We generate nine different realistically sized PIs to evaluate the performance of our model in large-scale applications. We first state our base scenario.

\subsubsection{Base Scenario}

We consider a strategic scenario in which a country's land forces are attacking enemy forces. Generally, land forces of a country consist of several armies, corps, brigades, and battalions. Figure 3 provides information on the organization of a land force.

The number of soldiers in an army can vary significantly between countries, commonly from 100,000 up to 200,000 or more. A corps typically includes from 20,000 to 50,000 soldiers. Under the current doctrine of most countries' land forces, armies are mostly concerned with both administrative and institutional missions. Usually, a corps is the highest level of command that is concerned with operations on the battlefield.

Hence, in our base scenario, we consider a corps that is in an offense position to defeat enemy forces. The corps has four brigades. A brigade may have four or five battalions depending on the mission, enemy threat, etc. We assume that each brigade has five battalions. Therefore, there are 20 battalions in total. Logistics planners determine three (eight) potential locations for Fixed-TPs (Mobile-TPs) in the corps' control area. The layout of the corps on the battlefield can be seen in Fig. 4, which shows the potential locations of Fixed-TPs and Mobile-TPs and known locations of battalions. In addition, all distances are taken from actual highway maps. The corps' transportation unit has eight commercial and 16 ammo trucks.

To reduce complexity, logistics planners group ammo into three groups according to their daily usage amounts. The high (medium, low) density group consists of the most (less frequently, least) used ammo types, such as infantry rifle bullets (anti-tank missiles, anti-aircraft missiles). To be supplied, all brigades cannot halt at the same time when they are engaged with the enemy. Hence, logistics planners decide to supply brigades in turn, such that battalions of a brigade have the

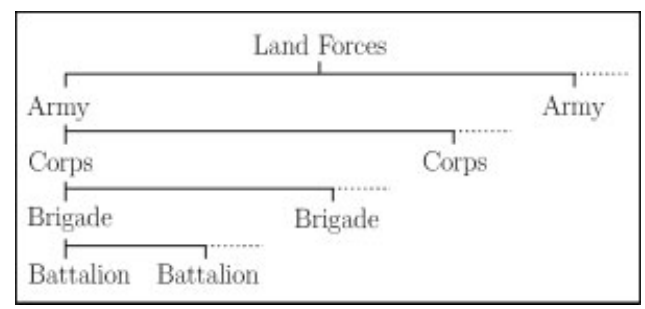

Figure 3. Organization of a representative land force.

Naval Research Logistics DOI 10.1002/nav 


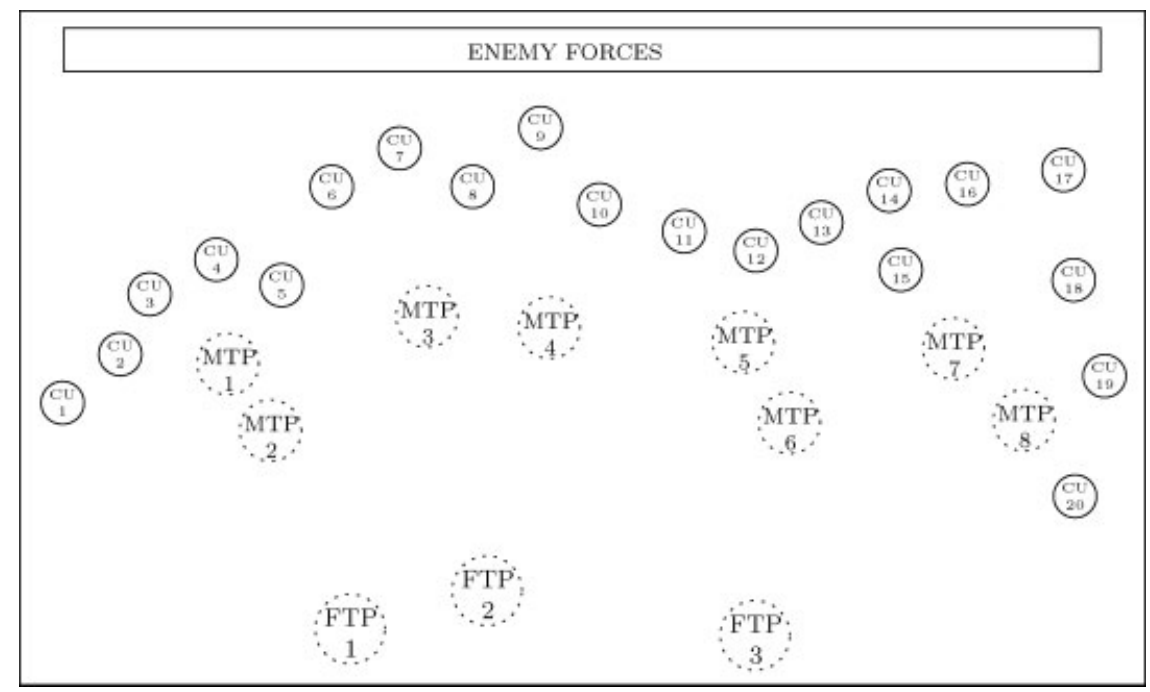

Figure 4. The corps' layout plan on the battlefield.

same supply time window, and brigades have nonoverlaping time windows.

To make the scenario more realistic, we must consider some specific military command and control structures and hierarchy among military units that always exist in real life. Therefore, we state three military requirements.

1. MILITARY REQUIREMENT 1: Because of the shortage of manpower or equipment resources and enemy threat, a corps cannot establish an unlimited number of Fixed-TPs in its control area. It is preferable to open one Fixed-TP per corps.

2. MILITARY REQUIREMENT 2: Every brigade uses a separate wireless communication channel to communicate with its battalions on the battlefield. Hence, it is always easier for a brigade to communicate with its own battalion. Besides, a battalion always reports to its own brigade about its location and demands. Thus, it is usually preferable to supply a combat unit from a Mobile-TP of its own brigade.

3. MILITARY REQUIREMENT 3: For the same reason stated in military requirement 1 , and because of military requirement 2 , it is preferable to open one Mobile-TP per brigade.

We need to state here that these military requirements result in a problem in which facility capacities don't have any role. However, we opt to keep the capacity constraints and give the model in its most general form to show that our model could be used in almost any (military or not) distribution system and to help readers compare our model with the existing network design or LRP models.

\subsubsection{Problem Instances}

We design nine different PIs of the base scenario by considering several combinations of two problem parameters as follows. We consider three different levels of ammo type numbers, that is $|P|=3,4,5$. We also consider three different levels of truck usage percentages to test the performance of the model against the number of both available and used trucks. These percentages are $75 \%, 50 \%$, and $83 \%$. At the $75 \%$ level, there exist eight commercial and 16 ammo trucks, out of which at least six commercial and 12 ammo trucks must be used. At the 50\% (83\%) level, there exist 12 commercial and 24 ammo trucks, out of which at least six (10) commercial and 12 (20) ammo trucks must be used. Table 5 exhibits the details of PIs and the computational results with both objective functions. Abbreviations $\mathrm{m}$ and $\mathrm{h}$ stand for minutes and hours.

Note that we use VI 2, strong branching and best-estimate search; solve LP relaxations at each node by primal simplex with devex pricing; only generate implied bounds, cover cuts, and clique cuts; implement aggressive scaling; and perform presolve at nodes.

As can be derived from the table, after $24 \mathrm{~h}$, the average gaps with the first objective are $2.8 \%, 4.8 \%$, and $6.6 \%$ for $|P|=3,4$, and 5, respectively. In addition, they are $2.4 \%$, $4.2 \%$, and $7.6 \%$ for $75 \%, 50 \%$, and $83 \%$ truck usage levels, respectively. It is clear that the problem gets harder to solve as the number of ammo types and the number of available and used trucks increase. The same observation holds for the second objective.

Table 6 shows the average percentages of fixed and variable costs in the total cost of the above PIs. Truck acquisition costs have the largest share with both objectives. Because 
Table 5. Computational results for the realistically sized problem instances.

\begin{tabular}{|c|c|c|c|c|c|c|c|c|c|c|c|c|}
\hline & \multirow[b]{2}{*}{ Obj. } & \multirow[b]{2}{*}{$|P|$} & \multirow[b]{2}{*}{ Truck usage (\%) } & \multicolumn{9}{|c|}{ Gaps (\%) after } \\
\hline & & & & $2 \mathrm{~m}$ & $8 \mathrm{~m}$ & $30 \mathrm{~m}$ & $1 \mathrm{~h}$ & $2 \mathrm{~h}$ & $4 \mathrm{~h}$ & $5 \mathrm{~h}$ & $10 \mathrm{~h}$ & $24 \mathrm{~h}$ \\
\hline PI A & $z 1$ & 3 & 75 & 12.04 & 10.75 & 10.16 & 4.98 & 1.90 & 1.34 & 1.15 & 0.93 & 0.89 \\
\hline PI B & & & 50 & 15.72 & 15.34 & 12.86 & 10.51 & 6.79 & 4.07 & 3.00 & 1.97 & 0.91 \\
\hline PI C & & & 83 & - & - & 10.42 & 8.71 & 6.71 & 6.71 & 6.71 & 6.71 & 6.71 \\
\hline PI D & & 4 & 75 & - & 13.22 & 8.93 & 8.59 & 7.27 & 4.20 & 3.65 & 2.98 & 2.45 \\
\hline PI E & & & 50 & - & - & - & - & 14.26 & 6.78 & 5.73 & 3.24 & 2.74 \\
\hline PI F & & & 83 & - & - & - & - & - & 10.58 & 10.36 & 10.07 & 9.26 \\
\hline PI G & & 5 & 75 & - & - & - & 11.39 & 10.29 & 5.28 & 4.72 & 4.24 & 3.94 \\
\hline PI H & & & 50 & - & - & 20.87 & 18.28 & 15.67 & 13.28 & 12.97 & 10.87 & 9.02 \\
\hline PI I & & & 83 & - & 20.83 & 16.12 & 14.03 & 10.67 & 6.83 & 6.69 & 6.69 & 6.69 \\
\hline PI A & $z 2$ & 3 & 75 & - & - & - & - & - & - & 0.48 & 0.44 & 0.44 \\
\hline PI B & & & 50 & 23.99 & 23.99 & 20.73 & 12.76 & 3.37 & 3.31 & 3.29 & 0.54 & 0.52 \\
\hline PI C & & & 83 & - & - & - & - & 9.31 & 9.16 & 9.16 & 8.98 & 8.96 \\
\hline PI D & & 4 & 75 & - & - & - & - & - & 3.72 & 1.07 & 1.02 & 0.58 \\
\hline PI E & & & 50 & - & - & 18.92 & 13.59 & 6.12 & 6.04 & 6.00 & 5.99 & 0.75 \\
\hline PI F & & & 83 & - & - & 11.03 & 9.06 & 9.04 & 9.00 & 8.98 & 8.87 & 8.87 \\
\hline PI G & & 5 & 75 & - & - & - & - & - & - & - & - & 0.57 \\
\hline PI H & & & 50 & - & - & - & - & 20.71 & 13.47 & 10.85 & 8.60 & 3.25 \\
\hline PI I & & & 83 & - & - & - & - & - & 9.20 & 9.16 & 9.11 & 8.88 \\
\hline
\end{tabular}

Mobile-TPs do not require substantial infrastructure investment, such as those required for underground storage facilities or bunkers, establishment costs are approximately half (one third at the $83 \%$ level) of the acquisition costs. Moreover, transportation costs are higher than the establishment costs with objective 1 .

Table 7 presents some general statistics on the model size. $|N|,|P|$, and $|V|$ are the numbers of nodes, ammo types, and vehicles in the problem instances. The other four statistics are obtained from the Model Statistics section of GAMS 22.3. Note that increasing the ammo type number does not cause a significant increase in the number of discrete variables. However, it increases the number of equations and non-zero coefficients in the problem matrix. It can also be seen that an increase in the number of vehicles increases the number of discrete variables, equations, and non-zero-elements.

We depict the solution of PI A in Fig. 5 as an example. As can be seen in the figure, one Fixed-TP and four Mobile-TPs are opened, and six commercial and 12 ammo trucks are dispatched. A cautious examination of the solution reveals that opening a Mobile-TP close to Fixed-TPs is almost always more cost-effective than opening one close to combat units. In fact, opening a Mobile-TP that is far from the front line combat units is also tactically advantageous. Such a transfer point would be less vulnerable to enemy fire and unexpected counter attacks. In addition, it would have more time to change its location in case of such contingencies.

\section{DYNAMIC MODEL DEVELOPMENT}

In this section, we formulate a dynamic Mobile-ADS design model assuming that known locations of combat units, as well as the set of potential Mobile-TP locations change in every consecutive planning period. As expected, solving such a mathematical model for a long planning period is computationally cumbersome. Hence, this section is rather intended to develop a dynamic model that should be used for advanced planning, when computational time is not an issue. Otherwise, we promote the successive use of our static model within the framework that is to be outlined in Subsection 6.1.

We assume that the planning horizon (combat duration) $T$ is partitioned into consecutive $24-\mathrm{h}$ time periods, represented by $t \in T$. In other words, there are $|T|$ time periods, i.e., $t \in\{1,2, \ldots,|T|\}$. We also assume that the potential location set for Fixed-TPs $\left(N_{\mathrm{F}}\right)$ does not change over time.

Table 6. Average percentages of fixed and variable costs in the total cost.

\begin{tabular}{|c|c|c|c|c|c|c|}
\hline \multirow[b]{2}{*}{$\begin{array}{c}\text { Truck usage } \\
\text { level }\end{array}$} & \multicolumn{3}{|c|}{ Objective $1(\%)$} & \multicolumn{3}{|c|}{ Objective $2(\%)$} \\
\hline & $\begin{array}{l}\text { Transfer point } \\
\text { establishment }\end{array}$ & $\begin{array}{c}\text { Truck } \\
\text { acquisition }\end{array}$ & $\begin{array}{c}\text { Ammo } \\
\text { transportation }\end{array}$ & $\begin{array}{l}\text { Transfer point } \\
\text { establishment }\end{array}$ & $\begin{array}{c}\text { Truck } \\
\text { acquisition }\end{array}$ & $\begin{array}{l}\text { Truck } \\
\text { driving }\end{array}$ \\
\hline $50-75 \%$ & 23 & 46 & 31 & 33 & 65 & 2 \\
\hline $83 \%$ & 18 & 59 & 23 & 23 & 75 & 2 \\
\hline
\end{tabular}


Table 7. General statistics on the model size of problem instances.

\begin{tabular}{lccccccc}
\hline & & & & $\begin{array}{c}\text { Non zero } \\
\text { elements }\end{array}$ & $\begin{array}{c}\text { Single } \\
\text { equations }\end{array}$ & $\begin{array}{c}\text { Single } \\
\text { variables }\end{array}$ & $\begin{array}{c}\text { Discrete } \\
\text { variables }\end{array}$ \\
\hline PI A & 31 & 3 & 24 & 240,513 & 27,996 & 81,493 & 11,623 \\
PI B-C & & & 36 & 358,217 & 40,596 & 121,257 & 16,679 \\
PI D & 4 & 24 & 306,767 & 34,402 & 104,464 & 12,123 \\
PI E-F & & & 36 & 456,751 & 49,754 & 155,388 & 17,179 \\
PI G & 5 & 24 & 373,021 & 40,808 & 127,435 & 12,623 \\
PI H-I & & 36 & 555,285 & 58,912 & 189,519 & 17,679 \\
\hline
\end{tabular}

$N_{\mathrm{M}}^{t}\left(N_{\mathrm{C}}^{t}\right)$ is the potential (known) location set of Mobile-TPs (combat units) in period $t \in T$.

We use the following set relations in the dynamic formulation: $N_{\mathrm{M}}^{t} \subseteq N_{\mathrm{M}}^{t+1}$ for all $t \in\{1,2, \ldots,|T|-1\}$. $N^{t}=N_{\mathrm{F}} \bigcup N_{\mathrm{M}}^{t} \bigcup N_{\mathrm{C}}^{t}, N_{\mathrm{FM}}^{t}=N_{\mathrm{F}} \bigcup N_{\mathrm{M}}^{t}, N_{\mathrm{MC}}^{t}=N_{\mathrm{M}}^{t} \bigcup N_{\mathrm{C}}^{t}$ for all $t \in T$ and $N_{\mathrm{FM}}=N_{\mathrm{F}} \bigcup N_{\mathrm{M}}^{|T|}$.

We permit the opening of new Mobile-TPs at the beginning of any time period and the closing of existing ones at the end of any time period. We also allow an existing Mobile-TP to re-open once it is closed or a new Mobile-TP to re-close once it is open. We assume that if a Mobile-TP is to be moved to another potential location, its transportation will take a relatively short time compared to the 24-h planning period; hence, we suppose that it changes its location instantaneously.

We know that the available number of trucks or the capacity of Mobile-TPs may vary between the time periods due to breakdowns, enemy fire, etc. However, we admit timeindependent fleet size and transfer point capacity for the sake of simplicity. Nevertheless, these parameters could easily be made time-dependent.

Note that in the dynamic formulation, we use the same decision variables as in the static model with an extra index $t \in T$, and they all refer to the related time period $t$. We also have two new binary decision variables: $y y_{i j}^{t}$ is 1 if Mobile-TP of a brigade is opened at potential location $i$ at the beginning of time period $t$ and relocated at a different potential location $j$ (such that $i$ and $j$ are potential locations of the same brigade) at the beginning of time period $t+1,0$ otherwise; and $x x_{\mathrm{v}}$ is 1 if vehicle $\mathrm{v}$ is dispatched from a transfer point in at least one time period, 0 otherwise. The dynamic Mobile-ADS design problem can be formulated as follows:

$$
\begin{array}{r}
\min \sum_{i \in N_{\mathrm{FM}}} \mathrm{FC}_{i} \cdot y_{i}^{t}+\sum_{t \in T} \sum_{i \in N_{\mathrm{M}}^{t}} \sum_{j \in N_{\mathrm{M}}^{t}} \sum_{\mathrm{v} \in V_{\mathrm{M}}} \mathrm{DC}_{\mathrm{v}}^{t} \cdot \mathrm{TI}_{i j}^{t} \cdot y y_{i j}^{t} \\
+\sum_{\mathrm{v} \in V} \mathrm{VC}_{\mathrm{v}} \cdot x x_{\mathrm{v}}+\sum_{t \in T} \sum_{i \in N^{t}} \sum_{j \in N^{t}} \sum_{\mathrm{v} \in V} \sum_{p \in P} \mathrm{TC}_{\mathrm{vp}}^{t} \cdot \mathrm{TI}_{i j}^{t} \cdot f_{i j v p}^{t} \\
+\sum_{t \in T} \sum_{i \in N_{\mathrm{C}}^{t}} \sum_{j \in N_{\mathrm{M}}^{t}} \sum_{\mathrm{v} \in V_{\mathrm{M}}} \mathrm{DC}_{\mathrm{v}}^{t} \cdot \mathrm{TI}_{i j}^{t} \cdot x_{i j v}^{t}
\end{array}
$$

subject to

$$
\begin{aligned}
& \sum_{\mathrm{v} \in V_{\mathrm{F}}}\left(\sum_{\substack{j \in N_{\mathrm{FM}}^{t} \\
j \neq i}} f_{j i v p}^{t}-\sum_{\substack{j \in N_{\mathrm{M}}^{t} \\
j \neq i}} f_{i j v p}^{t}\right) \\
& =\sum_{\mathrm{v} \in V_{\mathrm{M}}} \sum_{j \in N_{\mathrm{C}}^{t}} f_{i j v p}^{t} \forall i \in N_{\mathrm{M}}^{t}, p \in P, t \in T \\
& \sum_{\mathrm{v} \in V_{\mathrm{M}}}\left(\sum_{\substack{j \in N_{\mathrm{MC}}^{t} \\
j \neq i}} f_{j i v p}^{t}-\sum_{\substack{j \in N_{\mathrm{C}}^{t} \\
j \neq i}} f_{i j v p}^{t}\right) \\
& =Q_{i p}^{t} \quad \forall i \in N_{\mathrm{C}}^{t}, p \in P, t \in T
\end{aligned}
$$

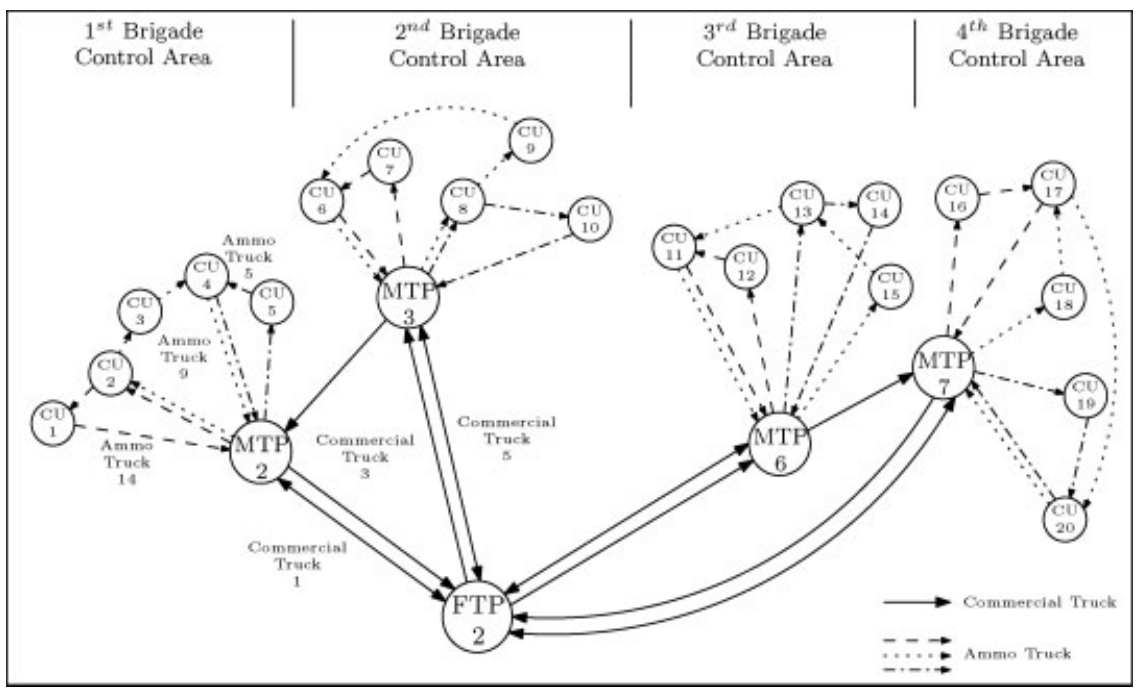

Figure 5. Solution of the base scenario. 
$\sum_{\substack{j \in N_{\mathrm{FM}}^{t} \\ j \neq i}} f_{j i v p}^{t} \geq \sum_{\substack{j \in N_{\mathrm{M}}^{t} \\ j \neq i}} f_{i j v p}^{t} \quad \forall i \in N_{\mathrm{M}}^{t}, \mathrm{v} \in V_{\mathrm{F}}, p \in P, t \in T$
$\sum_{\substack{j \in N_{\mathrm{MC}}^{t} \\ j \neq i}} f_{j i v p}^{t} \geq \sum_{\substack{j \in N_{\mathrm{C}}^{t} \\ j \neq i}} f_{i j v p}^{t} \quad \forall i \in N_{\mathrm{C}}^{t}, \mathrm{v} \in V_{\mathrm{M}}, p \in P, t \in T$

$\sum_{i \in N_{\mathrm{F}}} \sum_{j \in N_{\mathrm{M}}^{t}} x_{i j v}^{t} \leq 1 \quad \forall \mathrm{v} \in V_{\mathrm{F}}, t \in T$

$\sum_{i \in N_{\mathrm{M}}^{t}} \sum_{j \in N_{\mathrm{C}}^{t}} x_{i j v}^{t} \leq 1 \quad \forall \mathrm{v} \in V_{\mathrm{M}}, t \in T$

$\sum_{j \in N_{\mathrm{M}}^{t}} x_{j i v}^{t}=\sum_{j \in N_{\mathrm{M}}^{t}} x_{i j v}^{t} \quad \forall i \in N_{\mathrm{F}}, \mathrm{v} \in V_{\mathrm{F}}, t \in T$

(D-4a)

$\sum_{j \in N_{\mathrm{C}}^{t}} x_{j i v}^{t}=\sum_{j \in N_{\mathrm{C}}^{t+1}} x_{i j v}^{t+1}$

$\forall i \in N_{\mathrm{M}}^{t+1}, \mathrm{v} \in V_{\mathrm{M}}, t \in\{1,2, \ldots,|T|-1\}$

(D-4b-1)

$\sum_{j \in N_{\mathrm{C}}^{t}} x_{j i v}^{t}=\sum_{j \in N_{\mathrm{C}}^{t}} x_{i j v}^{t} \quad \forall i \in N_{\mathrm{M}}^{t}, \mathrm{v} \in V_{\mathrm{M}}, t=|T|$

$\sum_{\substack{j \in N_{\mathrm{FM}}^{t} \\ j \neq i}} x_{j i v}^{t}=\sum_{\substack{j \in N_{\mathrm{FM}}^{t} \\ j \neq i}} x_{i j v}^{t} \quad \forall i \in N_{\mathrm{M}}^{t}, \mathrm{v} \in V_{\mathrm{F}}, t \in T$

$\sum_{\substack{j \in N_{\mathrm{MC}}^{t} \\ j \neq i}} x_{j i v}^{t}=\sum_{\substack{j \in N_{\mathrm{MC}}^{t} \\ j \neq i}} x_{i j v}^{t} \quad \forall i \in N_{\mathrm{C}}^{t}, \mathrm{v} \in V_{\mathrm{M}}, t \in T$

$\sum_{\mathrm{v} \in V_{\mathrm{F}}} \sum_{j \in N_{\mathrm{M}}^{t}} f_{i j v p}^{t} \leq \mathrm{CD}_{i p} \cdot y_{i}^{t} \quad \forall i \in N_{\mathrm{F}}, p \in P, t \in T$

(D-6a)

$\sum_{\mathrm{v} \in V_{\mathrm{M}}} \sum_{j \in N_{\mathrm{C}}^{t}} f_{i j v p}^{t} \leq \mathrm{CD}_{i p} \cdot y_{i}^{t} \quad \forall i \in N_{\mathrm{M}}^{t}, p \in P, t \in T$

(D-6b)

$$
\begin{aligned}
\sum_{\mathrm{v} \in V_{\mathrm{F}}} \sum_{j \in N_{\mathrm{M}}^{t}} f_{i j v p}^{t} \leq & \left(\sum_{l \in N_{\mathrm{C}}^{t}} Q_{l p}^{t}\right) \cdot y_{i}^{t} \\
& \forall i \in N_{\mathrm{M}}^{t}, i \neq j, p \in P, t \in T
\end{aligned}
$$

$(\mathrm{D}-6 \mathrm{c})$

$$
f_{i j v p}^{t} \leq \mathrm{CV}_{\mathrm{vp}} \cdot x_{i j v}^{t}
$$

$\forall i \in N_{\mathrm{F}}, j \in N_{\mathrm{M}}^{t}, \mathrm{v} \in V_{\mathrm{F}}, p \in P, t \in T ;$

$\forall i, j \in N_{\mathrm{M}}^{t}, i \neq j, \mathrm{v} \in V_{\mathrm{F}}, p \in P, t \in T$;

$\forall i \in N_{\mathrm{M}}^{t}, j \in N_{\mathrm{C}}^{t}, \mathrm{v} \in V_{\mathrm{M}}, p \in P, t \in T$;

$\forall i, j \in N_{\mathrm{C}}^{t}, i \neq j, \mathrm{v} \in V_{\mathrm{M}}, p \in P, t \in T$

(D-7)

$$
\sum_{p \in P} f_{i j v p}^{t} \leq \mathrm{CT}_{\mathrm{v}} \cdot x_{i j v}^{t}
$$

$\forall i \in N_{\mathrm{F}}, j \in N_{\mathrm{M}}^{t}, \mathrm{v} \in V_{\mathrm{F}}, t \in T ;$

$\forall i, j \in N_{\mathrm{M}}^{t}, i \neq j, \mathrm{v} \in V_{\mathrm{F}}, t \in T$;

$\forall i \in N_{\mathrm{M}}^{t}, j \in N_{\mathrm{C}}^{t}, \mathrm{v} \in V_{\mathrm{M}}, t \in T$;

$\forall i, j \in N_{\mathrm{C}}^{t}, i \neq j, \mathrm{v} \in V_{\mathrm{M}}, t \in T$

$$
\sum_{p \in P} f_{i j v p}^{t} \geq x_{i j v}^{t}
$$

$\forall i \in N_{\mathrm{F}}, j \in N_{\mathrm{M}}^{t}, \mathrm{v} \in V_{\mathrm{F}}, t \in T ;$

$\forall i, j \in N_{\mathrm{M}}^{t}, i \neq j, \mathrm{v} \in V_{\mathrm{F}}, t \in T$;

$\forall i \in N_{\mathrm{M}}^{t}, j \in N_{\mathrm{C}}^{t}, \mathrm{v} \in V_{\mathrm{M}}, t \in T$;

$\forall i, j \in N_{\mathrm{C}}^{t}, i \neq j, \mathrm{v} \in V_{\mathrm{M}}, t \in T$

$$
\left(\sum_{l \in N_{\mathrm{C}}^{t}} Q_{l p}^{t}\right) \cdot w_{i j p}^{t} \geq f_{i j v p}^{t}
$$

$\forall i \in N_{\mathrm{F}}, j \in N_{\mathrm{M}}^{t}, \mathrm{v} \in V_{\mathrm{F}}, p \in P, t \in T ;$

$\forall i, j \in N_{\mathrm{M}}^{t}, i \neq j, \mathrm{v} \in V_{\mathrm{F}}, p \in P, t \in T$;

$\forall i \in N_{\mathrm{M}}^{t}, j \in N_{\mathrm{C}}^{t}, \mathrm{v} \in V_{\mathrm{M}}, p \in P, t \in T$;

$\forall i, j \in N_{\mathrm{C}}^{t}, i \neq j, \mathrm{v} \in V_{\mathrm{M}}, p \in P, t \in T$

$$
\begin{aligned}
& \sum_{\mathrm{v} \in V_{\mathrm{F}}} f_{i j v p}^{t} \geq w_{i j p}^{t} \\
& \forall i \in N_{\mathrm{F}}, j \in N_{\mathrm{M}}^{t}, p \in P, t \in T ; \\
& \forall i, j \in N_{\mathrm{M}}^{t}, i \neq j, p \in P, t \in T
\end{aligned}
$$

$\sum_{\mathrm{v} \in V_{\mathrm{M}}} f_{i j v p}^{t} \geq w_{i j p}^{t}$

$\forall i \in N_{\mathrm{M}}^{t}, j \in N_{\mathrm{C}}^{t}, p \in P, t \in T$
$\forall i, j \in N_{\mathrm{C}}^{t}, i \neq j, p \in P, t \in T$

$\mathrm{TE}_{i p}^{t} \leq \mathrm{tp}_{i p}^{t} \leq \mathrm{TL}_{i p}^{t} \quad \forall i \in N_{\mathrm{C}}^{t}, p \in P, t \in T$

$$
\operatorname{tp}_{i p}^{t}=0 \quad \forall i \in N_{\mathrm{F}}, p \in P, t \in T
$$

$$
\begin{aligned}
\operatorname{tp}_{i p}^{t}+ & \mathrm{TI}_{i j}^{t} \cdot w_{i j p}^{t}-\mathrm{TM}_{p}^{t} \cdot\left(1-w_{i j p}^{t}\right) \leq \mathrm{tp}_{j p}^{t} \\
& \forall i \in N_{\mathrm{F}}, j \in N_{\mathrm{M}}^{t}, p \in P, t \in T \\
& \forall i, j \in N_{\mathrm{M}}^{t}, i \neq j, p \in P, t \in T \\
& \forall i \in N_{\mathrm{M}}^{t}, j \in N_{\mathrm{C}}^{t}, p \in P, t \in T \\
& \forall i, j \in N_{\mathrm{C}}^{t}, i \neq j, p \in P, t \in T \\
\mathrm{tv}_{i v}^{t}+ & \mathrm{TI}_{i j}^{t} \cdot x_{i j v}^{t}-\mathrm{TM}^{t} \cdot\left(1-x_{i j v}^{t}\right) \leq \mathrm{tv}_{j v}^{t} \\
& \forall i \in N_{\mathrm{F}}, j \in N_{\mathrm{M}}^{t}, \mathrm{v} \in V_{\mathrm{F}}, t \in T \\
& \forall i, j \in N_{\mathrm{M}}^{t}, i \neq j, \mathrm{v} \in V_{\mathrm{F}}, t \in T \\
& \forall i \in N_{\mathrm{M}}^{t}, j \in N_{\mathrm{C}}^{t}, \mathrm{v} \in V_{\mathrm{M}}, t \in T \\
& \forall i, j \in N_{\mathrm{C}}^{t}, i \neq j, \mathrm{v} \in V_{\mathrm{M}}, t \in T
\end{aligned}
$$




$$
\begin{aligned}
2 \cdot y y_{i j}^{t} & \leq y_{i}^{t}+y_{j}^{t+1} \\
\forall i & \in N_{\mathrm{M}}^{t}, j \in N_{\mathrm{M}}^{t+1}, i \neq j, t \in\{1,2, \ldots,|T|-1\} \\
1+y y_{i j}^{t} & \geq y_{i}^{t}+y_{j}^{t+1} \\
\forall i & \in N_{\mathrm{M}}^{t}, j \in N_{\mathrm{M}}^{t+1}, i \neq j, t \in\{1,2, \ldots,|T|-1\}
\end{aligned}
$$

$x x_{\mathrm{v}} \geq x_{i j v}^{t} \quad \forall i \in N_{\mathrm{F}}, j \in N_{\mathrm{M}}^{t}, \mathrm{v} \in V_{\mathrm{F}}, t \in T$

$x x_{\mathrm{v}} \geq x_{i j v}^{t} \quad \forall i \in N_{\mathrm{M}}^{t}, j \in N_{\mathrm{C}}^{t}, \mathrm{v} \in V_{\mathrm{M}}, t \in T$

$f, \mathrm{tp}, \mathrm{tv}$ non-negative; $y, x, w, y y, x x$ binary.

The objective function contains five different cost components. The first component calculates the fixed cost of opening a new transfer point in Period 1 . Note that if we buy the equipment for a transfer point once, we can use it several times in different time periods. Opening a new transfer point actually means a transfer point is changing its location. Hence, in reality, the cost of opening a new transfer point is its cost of repositioning and this constitutes the second component. The third and fourth components are vehicle acquisition and ammunition distribution costs. The fifth component calculates the driving cost of empty trucks returning to their home transfer points.

Constraints have the same equation numbers (with a $\mathrm{D}$ in front that represents their dynamic version) as their duplicates in the static formulation. For example, constraints (D-1a) are the dynamic version of constraints (1a). However, constraints (D-4b-1) and (D-4b-2) are different from their static duplicates. To be exact, their static version is constraints (4b), which force each ammo truck to turn back to its home Mobile-TP from where it is dispatched. Nevertheless, in the dynamic model, Mobile-TPs can change their location in every consecutive time period.

Suppose a Mobile-TP is located at node $i$ in time period $t$ and dispatches ammo truck $v$. Then, that transfer point moves to node $j$ in period $t+1$, meaning that any truck that will serve combat units in period $t+1$ will be dispatched from node $j$. Consider that unit $k$ is the last unit on the route of truck $\mathrm{v}$ in period $t$. As depicted in Fig. 6, we must allow the truck to return to the transfer point, which may be located at a different node, of the next period (dashed line incoming to node $j$ ), as well as allowing it to return to the original transfer point (dashed line incoming to node $i$ ). Hence, we introduce constraints (D-4b-1) and (D-4b-2) in place of (4b) in the dynamic model. We assume that each truck returns to its home transfer point in time to be available the next day.

We also have four new constraints that are (D-16), (D-17), (D-18a), and (D-18b). Constraints (D-16) and (D-17) provide that if a transfer point changes its location in a time period,

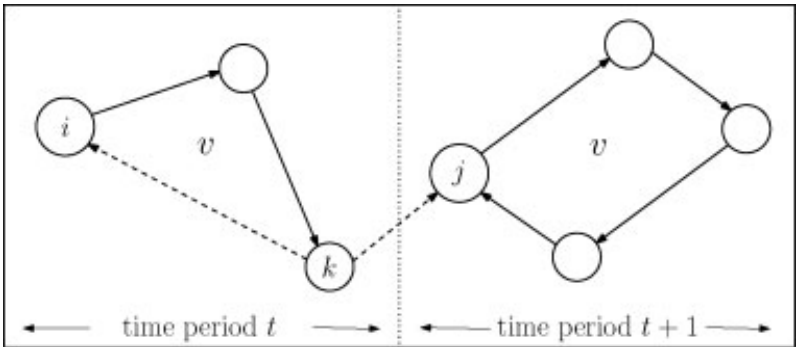

Figure 6. Returning arcs of ammo trucks to transfer points.

the cost of repositioning is properly added to the overall cost. Constraints (D-18a) and (D-18b) let us count the cost of a truck only once, even if it is used many times.

Remember that we partition the planning horizon into consecutive 24-h time periods. Since we have short planning periods, we assume that costs (vehicle acquisition and ammo distribution) do not vary from day to day. However, one may need to convert fixed and variable costs to some base present-value year in a multi-period model that uses longer planning periods, such as years. For more information on the use of discounting for multi-period military planning models, see [18].

We design three different PIs to assess the performance of the dynamic model for a planning horizon of two days. We again consider a corps that is in an offense position. In each day, the corps has four brigades and each brigade has five battalions to supply. Hence, there are 40 known battalion locations and 16 potential Mobile-TP locations in total. There are three potential locations for Fixed-TPs, which do not change over time. Figure 7 shows the layout of the corps on the battlefield for the two consecutive planning periods, i.e., $t^{1}$ and $t^{2}$. $\mathrm{CU} i^{1}\left(\mathrm{CU} i^{2}\right)$ represents the known location of combat unit $i$ in the first (second) period. Potential Mobile-TP locations for the first brigade in the first period are MTP1 and MTP2, whereas they are MTP1, MTP2, MTP9, and MTP10 in the second period.

We consider three different levels of ammo type numbers, that is $|P|=2,3,4$, in each PI. The corps' transportation unit has five commercial and 10 ammo trucks, out of which four commercial and eight ammo trucks are used. Table 8 presents the computational results. Table 9 gives some general statistics on the model size. As in the static model, an increase in the number of ammo types does not cause a significant increase in the number of discrete variables. However, it increases the number of other statistics.

We present the solution of PI $\mathrm{J}$ for the first period in Fig. 8. Solid (dashed and dotted) lines represent the routes of commercial (ammo) trucks. As can be seen in the figure, for the third brigade, a Mobile-TP is established at location MTP6 in Period 1, and it is moved to location MTP13 in Period 2. Hence, at the end of their routes, all ammo trucks return to 


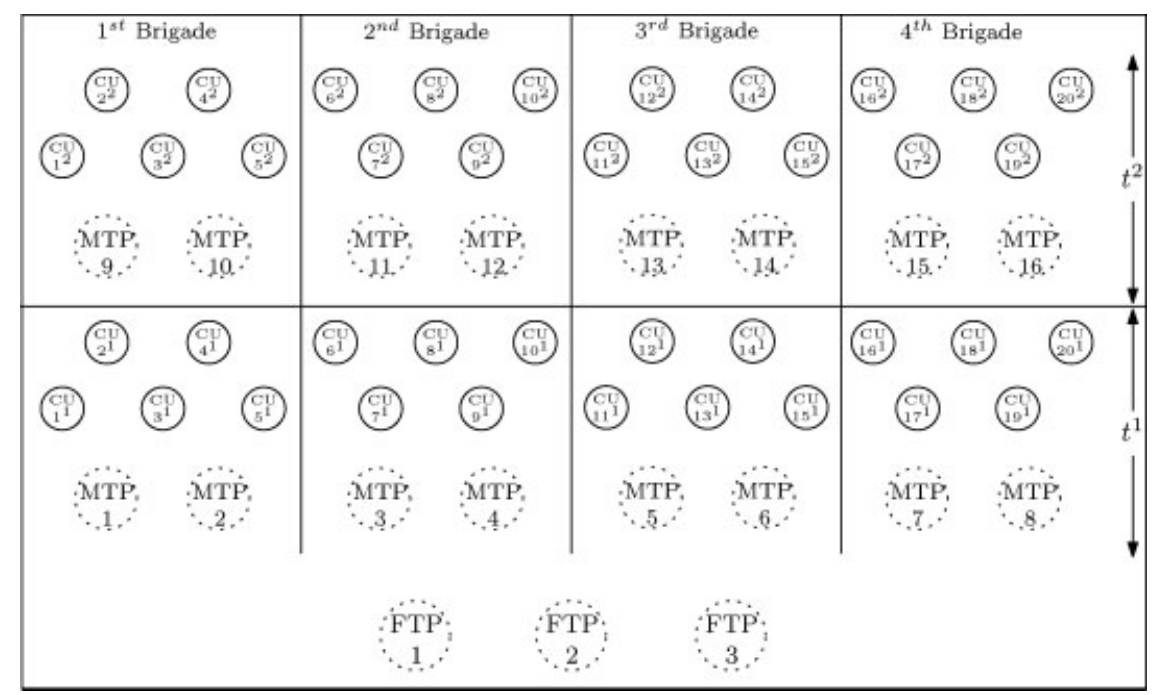

Figure 7. The corps' layout plan on the battlefield for two periods.

location MTP13 rather than MTP6. However, a Mobile-TP serves the second brigade at the same location, namely MTP3, in both periods. Thus, all ammo trucks return to the same location, MTP3, from where they were dispatched. Fig. 9 shows the solution for Period 2, and all ammo trucks return to their home transfer points, since this is the last planning period.

\section{STATIC MODEL IN REAL LIFE COMBAT SITUATIONS}

In this section, we first show how the static model could assist in a multi-day combat operation and then discuss how the model can help the logistics planners when faced with unplanned combat situations.

\subsection{Multi-Period Planning with the Static Model}

In what follows, we provide a framework to guide in the successive use of the static model for multi-period strategic decision making. Recall that one of the main differences of the two models lies in the costing structure of the ammo trucks mainly due to the fact that in the dynamic model this

Table 8. Computational results for the multi-period problem instances.

\begin{tabular}{lccccccccc}
\hline & \multicolumn{10}{c}{ Gaps (\%) after } \\
\cline { 2 - 10 } & $1 \mathrm{~h}$ & $6 \mathrm{~h}$ & $7 \mathrm{~h}$ & $8 \mathrm{~h}$ & $9 \mathrm{~h}$ & $10 \mathrm{~h}$ & $15 \mathrm{~h}$ & $20 \mathrm{~h}$ & $24 \mathrm{~h}$ \\
\hline PI J & - & - & - & 23.38 & 23.25 & 23.23 & 23.23 & 23.12 & 23.04 \\
PI K & - & - & 27.99 & 27.96 & 27.89 & 28.31 & 27.54 & 27.44 & 27.41 \\
PIL & - & 26.34 & 26.18 & 26.17 & 26.14 & 26.13 & 25.03 & 24.95 & 24.91 \\
\hline
\end{tabular}

cost refers to the travel cost to the new (possible) Mobile-TP location whereas in the static model this cost is neglected. To compare the two models more fairly, we now include the Mobile-TP return cost within the objective function of the static model. As another modification, we adjust the fixed costs of Mobile-TPs in the static model in compliance with the logic we used for the dynamic model. Note that the first component of the objective function of the dynamic model corresponds to the fixed cost of opening a new transfer point in Period 1. Since we incur this cost only once in the first period, in the consecutive periods, we take the fixed costs of all opened Mobile-TPs as zero and add their repositioning costs (whenever applicable). Moreover, the potential MobileTP location set in each period should also contain the selected Mobile-TP locations of the previous period as output by the static model.

Consider the layout of the corps in Fig. 7. The static model can be used to solve this problem exactly in the same way explained in Section 3, with the above suggested adjustment. Figs. 10 and 11 present the static model solution for the first and the second periods. In a comparison with the dynamic solution we can see both similarities and differences. Briefly, both the dynamic and the static models keep MTP3 at the

Table 9. General statistics on the model size of the multi-period problem instances.

\begin{tabular}{lrrrrrrr}
\hline & & & & $\begin{array}{c}\text { Non zero } \\
\text { elements }\end{array}$ & $\begin{array}{c}\text { Single } \\
\text { equations }\end{array}$ & $\begin{array}{c}\text { Single } \\
\text { variables }\end{array}$ & $\begin{array}{c}\text { Discrete } \\
\text { variables }\end{array}$ \\
\hline PI J & 59 & 2 & 15 & 451,287 & 45,402 & 237,400 & 30,421 \\
PI K & & 3 & & 631,551 & 57,694 & 341,738 & 31,969 \\
PI L & 4 & & 811,815 & 69,986 & 446,076 & 33,517 \\
\hline
\end{tabular}

Naval Research Logistics DOI 10.1002/nav 


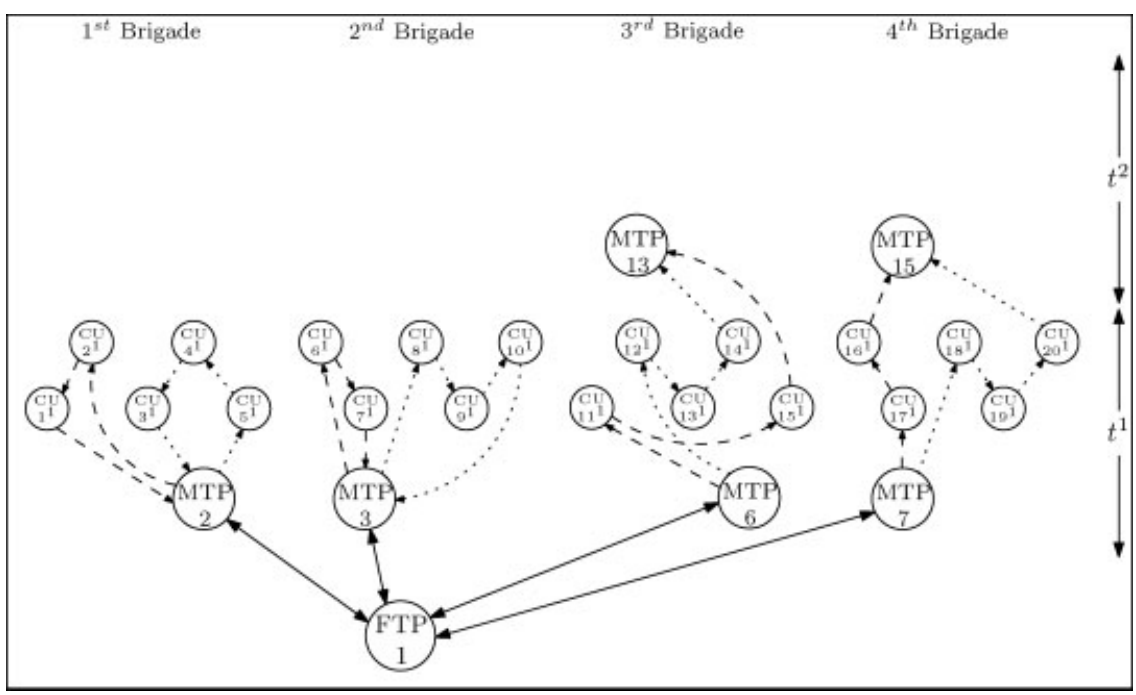

Figure 8. Dynamic model solution of the multi-period scenario for the first period.

same place for both periods and move MTP7 to MTP15 in the second period. However, the static model continues to use MTP6, but moves MTP2 to MTP10 in the second period, whereas the dynamic model continues to use MTP2 and relocates MTP6 to MTP13.

As for the truck routes, again there are similarities and differences between the solutions of the models. For example, ammo truck routes are similar for the second brigade in Period 1 and for the third and fourth brigades in Period 2. However, the most obvious difference is in commercial truck routes. The dynamic model uses four commercial trucks whereas the static model dispatches only three. At the same time, it can also clearly be seen from the figures that the dynamic model has less traffic than the static model, which in turn results in a lower objective value of 1164.6 versus 1498.8 of the static model.

Which model is more advantageous depends on the combat environment and enemy capabilities. For instance, as it is already remarked in Subsection 3.2 if the enemy has the ability to detect our logistics convoys then the dynamic model would be more advantageous, because it has less traffic.

\subsection{Facing Unplanned Contingencies with the Static Model}

Consider that logistics planners begin to execute the distribution plan that is seen in Figs. 8 and 9. However, just before the distribution plan for the second day is put into action,

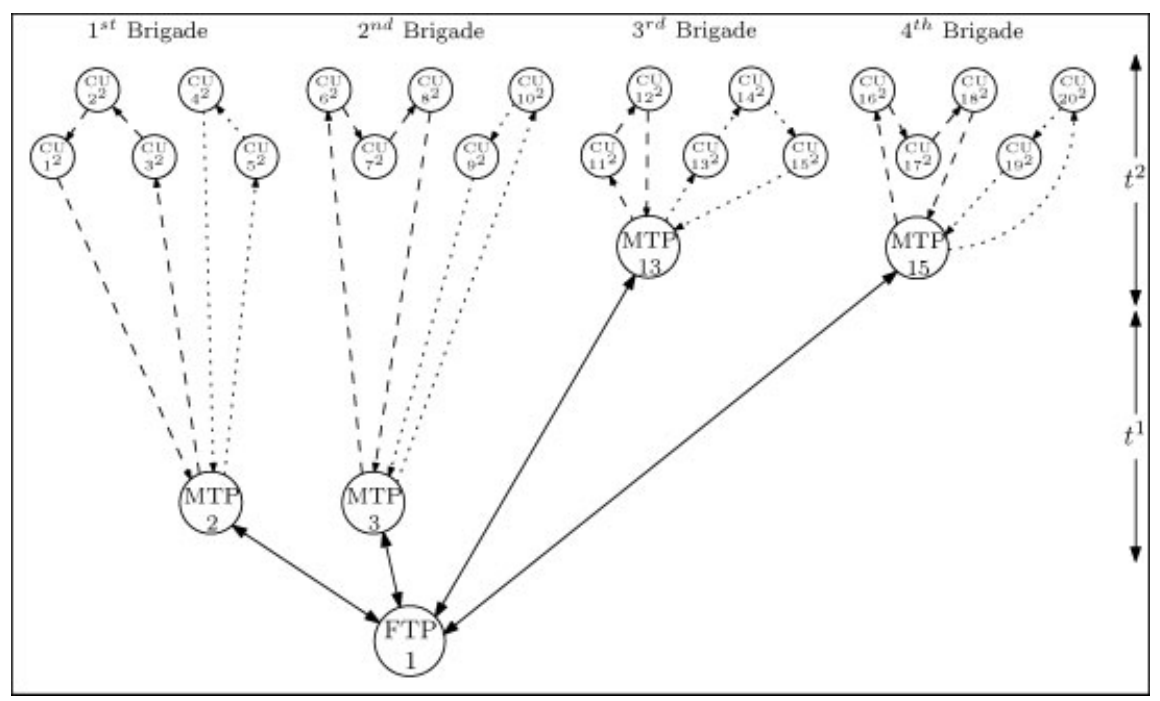

Figure 9. Dynamic model solution of the multi-period scenario for the second period. 


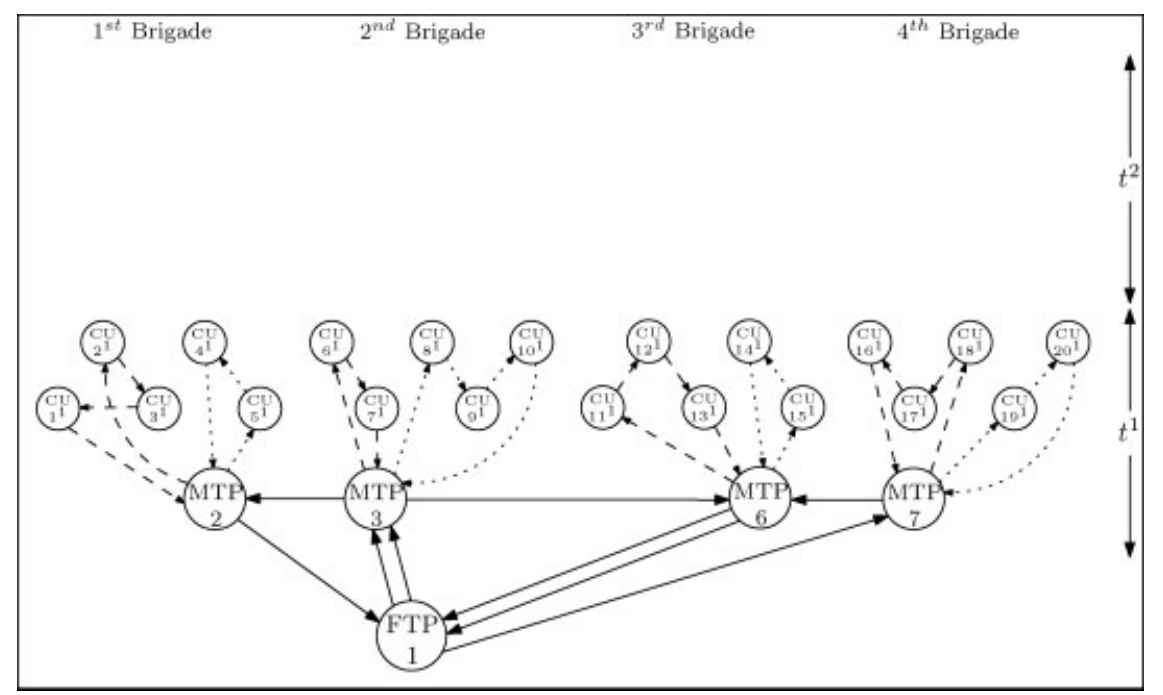

Figure 10. Static model solution of the multi-period scenario for the first period.

they receive the following Logistics Related Update from the Corps Headquarters: For the first brigade, due to unexpectedly high enemy presence, Battalion 4 triples its ammo requirements for each type. The bridge between MTP2 and Battalion 5 has been destroyed and other battalions of the brigade are too far away that Battalion 5 must be supplied by the second brigade (MTP3). For the second brigade, due to unexpectedly high enemy presence, Battalion 8 doubles its ammo requirements for each type. For the third brigade, everything is as planned and no change is required. For the fourth brigade, due to heavy enemy artillery fire, MTP15 is no longer suitable for any further logistics operations. All ammo trucks have been moved to MTP16 and distribution must be made from this site from now on. Transportation between Battalion 16 and 17 is blocked because of an enemy mine field.

Similar updates can be encountered at any (the first, the last, or a middle) day of a given distribution plan. In such circumstances, logistics planners must prepare the new distribution plan according to the update. However, the first and utmost important challenge is not to prepare the whole distribution plan. The real issue is to save the present day as soon and as good as possible, and then to prepare the distribution plan for the rest of the battle duration. For the present day, we have serious time pressure to answer the needs of the combat units confronting the enemy. But for the next days, we will

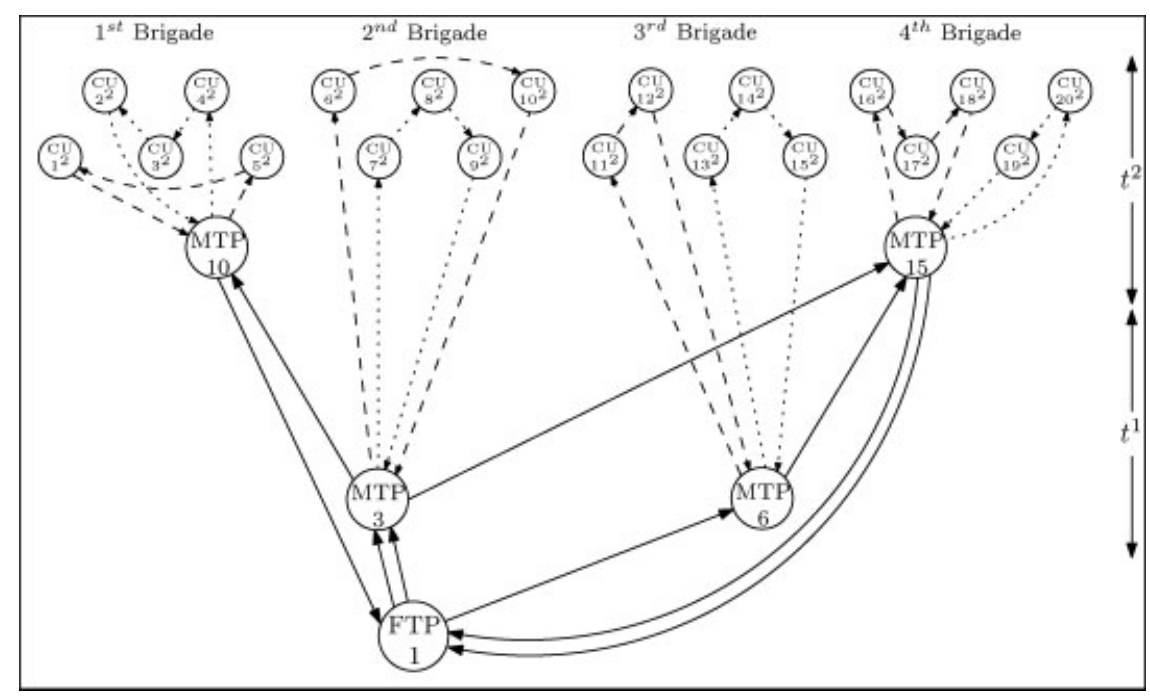

Figure 11. Static model solution of the multi-period scenario for the second period. 


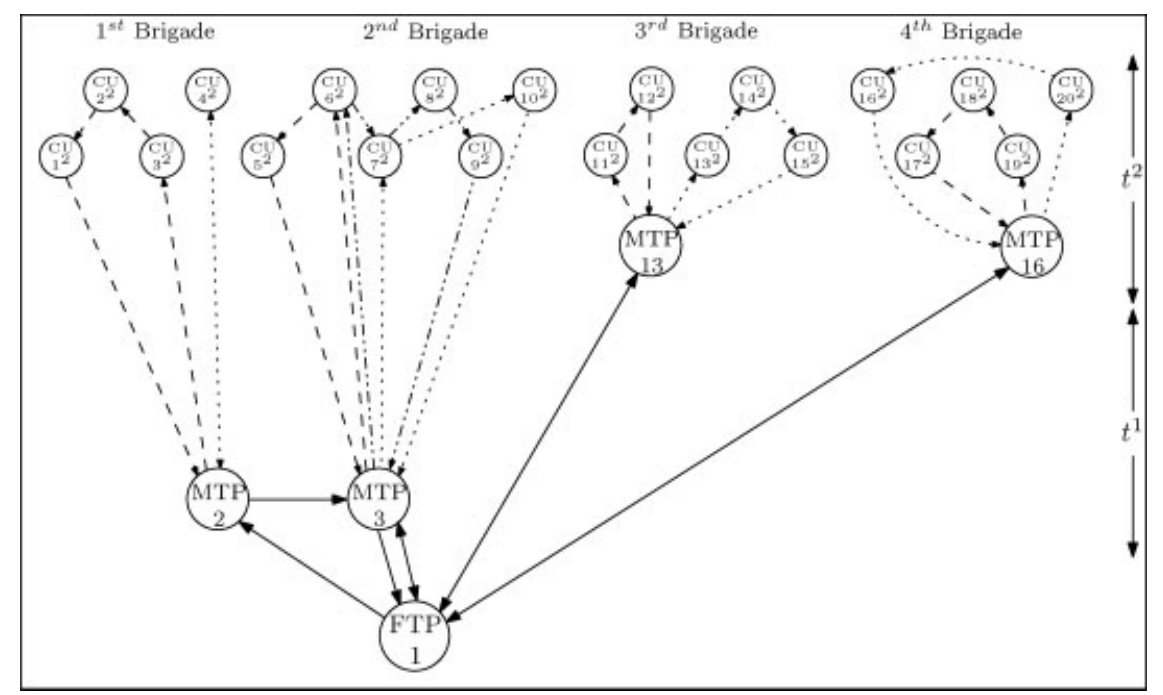

Figure 12. New solution for the second period.

have enough time to plan. Hence, the best way to proceed is to run the static model for the present day and then run the dynamic model for the rest of the combat duration.

Figure 12 shows the new distribution plan for the second period. This solution is obtained with the static model in $7 \mathrm{~m}$., which we believe is short enough to answer the needs of the combat units in time.

\section{SUMMARY AND CONCLUSIONS}

In this article, we develop the Mobile Ammunition Distribution System to provide an effective and flexible distribution system on the battlefield. We create a classification scheme consisting of 17 problem characteristics, and classify 78 previous articles. Then, we show that our problem includes some aspects that have received little attention so far. Hence, observing that the literature does not contain any model that can handle our problem, we first develop a static mixed integer programming formulation. We then derive several valid inequalities to lessen the solution time and solve nine problem instances, indicating that realistically sized problems can be solved satisfactorily well in a reasonable amount of time.

Next, to better capture the reality of the battlefield, we introduce a dynamic model that can help us design the distribution system for each day of the entire combat horizon. Then, we illustrate the use of the static model for strategic planning in which we have multiple time periods. Finally, we present how the static model can also be utilized when logistics planners are faced with spontaneous problems which must be solved in a short amount of time.

The Operations Plan (OPlan) of a battle must be closely coordinated with its Logistics Plan (LPlan). Otherwise, if LPlan does not support OPlan, the mission may fail. Thus, as one of the most important supply classes, the Ammo Distribution Supplement (ADistSupp) of a LPlan gains the utmost importance, as it contains various ammo depot locations, transportation routes, and resources to be used for transportation. Today, the ADistSupp of most countries' land forces is prepared manually, and we propose our mathematical model as an alternative in this process.

Logistics planners must assure the adequacy and feasibility of their plans. Our model not only provides a means to correctly prepare ADistSupp in compliance with the OPlan but also enables logistics planners to detect the bottlenecks, such as the inappropriate locations of transfer points and the insufficient number of ammo trucks. By using our model, logistics planners can determine vulnerabilities ahead of time, evaluate options, conduct what-if analyses, and help commanders adjust either the OPlan or LPlan by making recommendations about courses of action as follows: "Current ADistSupp cannot meet the requested time windows in the OPlan with this rate of operation tempo. We should either slow down the tempo or locate an additional Mobile-TP or provide 10 more ammo trucks."

Our model can also assist logistics planners to easily revise ADistSupp if unplanned contingencies arise once the battle commences. Such unplanned contingencies, scenario analyses, or alternative solution explorations, which typically arise within a single planning period (a day in this study) must be urgently answered during the associated period. In case of such events, our model is able to conduct scenario analyses to explore alternative solutions and to examine the trade-offs.

\section{ACKNOWLEDGMENTS}

This research is supported by the Turkish Academy of Sciences. 


\section{REFERENCES}

[1] D. Aksen and K. Altinkemer, "A location-routing problem for the conversion to the "click-and-mortar" retailing: The static case," Working paper, College of Administrative Science and Economics, Koc University, Istanbul, Turkey, 2005.

[2] S. Alumur and B.Y. Kara, A new model for the hazardous waste location-routing problem, Comput Oper Res 34 (2007), 1406-1423.

[3] D. Ambrosino, A. Sciomachen, and M.G. Scutella, A heuristic based on multi-exchange techniques for a regional fleet assignment location-routing problem, Comput Oper Res 36 (2009), $442-460$.

[4] D. Ambrosino and M.G. Scutellà, Distribution network design: New problems and related models, Eur J Oper Res 165 (2005), 610-624.

[5] I. Averbakh and O. Berman, Routing and location-routing $p$ delivery men problems on a path, Transport Sci 28 (1994), 162-166.

[6] I. Averbakh and O. Berman, Probabilistic sales-delivery man and sales-delivery facility location problems on a tree, Transport Sci 29 (1995), 184-197.

[7] I. Averbakh, O. Berman, and D. Simchi-Levi, Probabilistic a priori routing-location problems, N Res Lo 41 (1994), 973-989.

[8] S. Barreto, C. Ferreira, J. Paixao, and B.S. Santos, Using clustering analysis in a capacitated location-routing problem, Eur J Oper Res 179 (2007), 968-977.

[9] R.T. Berger, C.R. Coullard, and M.S. Daskin, Location-routing problems with distance constraints, Transport Sci 41 (2007), 29-43.

[10] O. Berman, P. Jaillet, and D. Simchi-Levi, "Location-routing problems with uncertainty," Facility location: a survey of applications and methods, Z. Drezner (Editor), SpringerVerlag Inc., New York, 1995, pp. 427-452.

[11] O. Berman and D. Simchi-Levi, Minisum location of a travelling salesman, Networks 16 (1986), 239-254.

[12] O. Berman and D. Simchi-Levi, Finding the optimal a priori tour and location of a traveling salesman with nonhomogeneous customers, Transport Sci 22 (1988), 148-154.

[13] D.J. Bertsimas, Traveling salesman facility location problems, Transport Sci 23 (1989), 184-191.

[14] A. Billionnet, S. Elloumi, and L.G. Djerbi, Designing radiomobile access networks based on synchronous digital hieratchy rings, Comput Oper Res 32 (2005), 379-394.

[15] J.H. Bookbinder and K.E. Reece, Vehicle routing considerations in distribution system design, Eur J Oper Res 37 (1988), 204-213.

[16] I.M. Branco and J.D. Coelho, The hamiltonian p-median problem, Eur J Oper Res 47 (1990), 86-95.

[17] A. Brooke, D. Kendrick, A. Meeraus, and R. Raman, GAMS: A users guide, GAMS Development Corporation, Washington, DC, 2005.

[18] G.G. Brown, R.F. Dell, and A.M. Newman, Optimizing military capital planning, Interfaces 34 (2004), 415-425.

[19] A. Bruns and A. Klose, An iterative heuristic for locationrouting problems based on clustering, Proceedings of the Second International Workshop on Distribution Logistics, Oegstgeest, The Netherlands, 1995.

[20] R.C. Burness and J.A. White, The travelling salesman location problem, Transport Sci 10 (1976), 348-360.

[21] R. Caballero, M. Gonzáles, F.M. Guerrero, J. Molina, and C. Paralera, Solving a multiobjective location routing problem with a metaheuristic based on tabu search, Application to a real case in Andalusia, Eur J Oper Res 177 (2007), 1751-1763.

[22] P. Cappanera, G. Gallo, and F. Maffioli, Discrete facility location and routing of obnoxious activities, Discr Appl Math 133 (2004), 3-28.

[23] A.W. Chan and R.L. Francis, A round-trip location problem on a tree graph, Transport Sci 10 (1976), 35-51.

[24] A.W. Chan and D.W. Hearn, A rectilinear distance round-trip location problem, Transport Sci 11 (1977), 107-123.

[25] Y. Chan, W.B. Carter, and M.D. Burnes, A multiple-depot, multiple-vehicle, location-routing problem with stochastically processed demands, Comput Oper Res 28 (2001), 803-826.

[26] T.W. Chien, Heuristic procedures for practical sized uncapacitated location capacitated routing problems, Decision Sci 24 (1993), 995-1021.

[27] C. Duhamel, P. Lacomme, C. Prins, and C. Prodhon, A memetic approach for the capacitated location routing problem, University of Technology of Troyes, France, October 23-24, 2008. EU/Meeting 2008.

[28] GAMS/Cplex, GAMS: The solver manuals, GAMS Development Corporation, Washington, DC, 2005.

[29] G. Ghiani and G. Laporte, Eulerian location problems, Networks 34 (1999), 291-302.

[30] I. Giannikos, A multiobjective programming model for locating treatment sites and routing hazardous waste, Eur J Oper Res 104 (1998), 333-342.

[31] NATO Logistics Handbook, Retrieved June 10, 2008. Available at: http://www.nato.int/docu/logi-en/1997/lo-405.htm.

[32] P.H. Hansen, B. Hegedahl, S. Hjortkjær, and B. Opel, A heuristic solution to the warehouse location-routing problem, Eur $\mathbf{J}$ Oper Res 76 (1994), 111-127.

[33] H. Harrison, A planning system for facilities and resources in distribution networks, Interfaces 9 (1979), 6-22.

[34] T.L. Jacobs and J.M. Warmerdam, Simultaneous routing and siting for hazardous-waste operations, J Urban Plann Dev 120 (1994), 115-131.

[35] S.K. Jacobsen and O.B.G. Madsen, A comparative study of heuristics for a two-level routing-location problem, Eur J Oper Res 5 (1980), 378-387.

[36] M. Jamil, R. Batta, and D.M. Malon, The traveling repairperson home base location problem, Transport Sci 28 (1994), $150-161$.

[37] W.K. Freeman, Jr., A study of ammunition consumption, Master's thesis, U.S. Army Command and General Staff College, Fort Leavenwarth, KS, United States, 2005.

[38] A. Kolen, The round-trip p-center and covering problem on a tree, Transport Sci 19 (1985), 222-234.

[39] M. Labbé, G. Laporte, I.R. Martín, and J.J.S. González, Locating median cycles in networks, Eur J Oper Res 160 (2005), 457-470.

[40] G. Laporte, "Location-routing problems," Vehicle routing: Methods and studies, B.L. Golden and A.A. Asad (Editors), North-Holland Publishing, Amsterdam, Holland, 1988, pp. 163-198.

[41] G. Laporte and P.J. Dejax, Dynamic location-routing problems, J Oper Res Soc 40 (1989), 471-482.

[42] G. Laporte, F. Louveaux, and H. Mercure, Models and exact solutions for a class of stochastic location-routing problems, Eur J Oper Res 39 (1989), 71-78.

[43] G. Laporte and Y. Nobert, An exact algorithm for minimizing routing and operating costs in depot location, Eur J Oper Res 6 (1981), 224-226. 
[44] G. Laporte, Y. Nobert, and D. Arpin, An exact algorithm for solving a capacitated location-routing problem, Ann Oper Res 6 (1986), 293-310.

[45] G. Laporte, Y. Nobert, and P. Pelletier, Hamiltonian location problems, Eur J Oper Res 12 (1983), 82-89.

[46] G. Laporte, Y. Nobert, and S. Taillefer, Solving a family of multi-depot vehicle routing and location-routing problems, Transport Sci 22 (1988), 161-172.

[47] Y. Lee, S. Kim, S. Lee, and K. Kang, A location-routing problem in designing optical internet access with wdm systems, Photonic Netw Commun 6 (2003), 151-160.

[48] L. Levy and L. Bodin, The arc oriented location routing problem, Informs 27 (1989), 74-94.

[49] C.K.Y. Lin, C.K. Chow, and A. Chen, A location-routingloading problem for bill delivery services, Comput Ind Eng 43 (2002), 5-25.

[50] C.K.Y. Lin and R.C.W. Kwok, Multi-objective metaheuristics for a location-routing problem with multiple use of vehicles on real data and simulated data, Eur J Oper Res 175 (2006), 1833-1849.

[51] G.F. List and P.B. Mirchandani, An integrated network/planar multiobjective model for routing and siting for hazardous materials and wastes, Transport Sci 25 (1991), 146-156.

[52] G.F. List, P.B. Mirchandani, M.A. Turnquist, and K.G. Zografos, Modeling and analysis for hazardous materials transportation: Risk analysis, routing/scheduling and facility location, Transport Sci 25 (1991), 100-114.

[53] S.C. Liu and S.B. Lee, A two-phase heuristic method for the multi-depot location routing problem taking inventory control decisions into consideration, Int J Adv Manuf Technol 22 (2003), 941-950.

[54] O.B.G. Madsen, Methods for solving combined two level location-routing problems of realistic dimensions, Eur J Oper Res 12 (1983), 295-301.

[55] J. Melechovsky, C. Prins, and R.W. Calvo, A metaheuristic to solve location-routing problem with non-linear costs, J Heuristics 11 (2005), 375-391.

[56] C.E. Miller, A.W. Tucker, and R.A. Zemlin, Integer programming formulation of traveling salesman problems, J Assoc Comput Mach 7 (1960), 326-329.

[57] H. Min, Consolidation terminal location-allocation and consolidated routing problems, J Bus Logistics 17 (1996), 235-263.

[58] H. Min, V. Jayaraman, and R. Srivastava, Combined locationrouting problems: A synthesis and future research directions, Eur J Oper Res 108 (1998), 1-15.

[59] K.G. Murty and P.A. Djang, The U.S. Army National Guard's mobile training simulators location and routing problem, Oper Res 47 (1999), 175-182.

[60] G. Nagy and S. Salhi, Nested heuristic methods for the location-routing problem, J Oper Res Soc 47 (1996), 1166-1174.

[61] G. Nagy and S. Salhi, A nested location-routing heuristic using route length estimation, Stud Locat Analysis 10 (1996), 109-127.

[62] G. Nagy and S. Salhi, The many-to-many location-routing problem, TOP 6 (1998), 261-275.

[63] G. Nagy and S. Salhi, Location-routing: Issues, models and methods, Eur J Oper Res 177 (2007), 649-672.
[64] J.M. Nambiar, L.F. Gelders, and L.N. Van Wassenhove, A large scale location-allocation problem in the natural rubber industry, Eur J Oper Res 6 (1981), 183-189.

[65] J.M. Nambiar, L.F. Gelders, and L.N. Van Wassenhove, Plant location and vehicle routing in the Malaysian rubber smallholder sector: A case study, Eur J Oper Res 38 (1989), 14-26.

[66] I. Or and W.P. Pierskalla, A transportation location-allocation model for regional blood banking, AIIE Trans 11 (1979), 86-95.

[67] Z. Ozyurt and D. Aksen, "Solving the multi-depot locationrouting problem with lagrangian relaxation," Extending the horizons: Advances in computing, optimization, and decision technologies, Vol. 37, E.K. Baker, A. Joseph, A. Mehrotra, and M.A. Trick (Editors), Springer, 2007, pp. 125-144.

[68] Wikipedia Home Page, Retrieved June 10, 2008. Available at: http://en.wikipedia.org/wiki/MainPage.

[69] Windows XP Home Page, Microsoft Corporation 2007, Retrieved September 16, 2007. Available at: http://www. microsoft.com/windows/products/windowsxp/default.mspx.

[70] J. Perl and M.S. Daskin, A unified warehouse location-routing methodology, J Bus Logistics 5 (1984), 92-111.

[71] J. Perl and M.S. Daskin, A warehouse location-routing problem, Transport Res B 19B (1985), 381-396.

[72] C. ReVelle, J. Cohon, and D. Shobrys, Simultaneous siting and routing in the disposal of hazardous wastes, Transport Sci 25 (1991), 138-145.

[73] S. Salhi and M. Fraser, An integrated heuristic approach for the combined location vehicle fleet mix problem, Stud Locat Analysis 8 (1996), 3-21.

[74] S. Salhi and G. Nagy, Consistency and robustness in locationrouting, Stud Locat Analysis 13 (1999), 3-19.

[75] S. Salhi and G.K. Rand, The effect of ignoring routes when locating depots, Eur J Oper Res 39 (1989), 150-156.

[76] M.A. Sambola, J.A. D.íaz, and E. Fernández, A compact model and tight bounds for a combined location-routing problem, Comput Oper Res 32 (2005), 407-428.

[77] M.A. Sambola, E. Fernández, and G. Laporte, Heuristic and lower bound for a stochastic location-routing problem, Eur J Oper Res 179 (2007), 940-955.

[78] M. Schwardt and J. Dethloff, Solving a continuous locationrouting problem by use of a self-organizing map, Int J Phys Distrib Logist Manag 35 (2005), 390-408.

[79] F. Semet, A two-phase algorithm for the partial accessibility constrained vehicle routing problem, Ann Oper Res 61 (1995), 45-65.

[80] F. Semet and E. Taillard, Solving real-life vehicle routing problems efficiently using tabu search, Ann Oper Res 41 (1993), 469-488.

[81] Z.J.M. Shen and L. Qi, Incorporating inventory and routing costs in strategic location models, Eur J Oper Res 179 (2007), 372-389.

[82] D. Simchi-Levi, The capacitated traveling salesman location problem, Transport Sci 25 (1991), 9-18.

[83] D. Simchi-Levi and O. Berman, A heuristic algorithm for the travelling salesman location problem on networks, Oper Res 66 (1988), 478-484.

[84] R. Srivastava, Alternate solution procedures for the locationrouting problem, Omega 21 (1993), 497-506.

[85] R. Srivastava and W.C. Benton, The location-routing problem: Considerations in physical distribution system design, Comput Oper Res 17 (1990), 427-435.

Naval Research Logistics DOI 10.1002/nav 
[86] C.L. Stowers and U.S. Palekar, Location models with routing considerations for a single obnoxious facility, Transport Sci 27 (1993), 350-362.

[87] D. Tuzun and L.I. Burke, A two-phase tabu search approach to the location routing problem, Eur J Oper Res 116 (1999), 87-99.

[88] M. Wasner and G. Zapfel, An integrated multi-depot hublocation vehicle routing model for network planning of parcel service, Int J Prod Econ 90 (2004), 403-419.
[89] T. Wu, C. Low, and J. Bai, Heuristic soluions to multidepot location-routing problems, Comput Oper Res 29 (2002), 1393-1415.

[90] W. Yi and L. Ozdamar, A dynamic logistics coordination model for evacuation and support in disaster response activities, Eur J Oper Res 179 (2007), 1177-1193.

[91] K.G. Zografos and S. Samara, Combined location-routing model for hazardous waste transportation and disposal, Transport Res Rec 1245 (1989), 52-59. 